\title{
ZFP91 disturbs metabolic fitness and antitumor activity of tumor-infiltrating $T$ cells
}

\author{
Feixiang Wang, ${ }^{1}$ Yuerong Zhang, ${ }^{1}$ Xiaoyan Yu, ${ }^{1}$ Xiao-Lu Teng, ${ }^{1}$ Rui Ding, ${ }^{1}$ Zhilin Hu, ${ }^{1}$ Aiting Wang, ${ }^{1}$ Zhengting Wang, ${ }^{2}$ \\ Youqiong Ye, ${ }^{1}$ and Qiang Zou' ${ }^{1}$ \\ 'Shanghai Institute of Immunology, Department of Immunology and Microbiology, State Key Laboratory of Oncogenes and Related Cenes, Hongqiao International Institute of Medicine, Tongren Hospital, \\ Shanghai Jiao Tong University School of Medicine, Shanghai, China. ²Department of Gastroenterology, Ruijin Hospital, Shanghai Jiao Tong University School of Medicine, Shanghai, China.
}

\begin{abstract}
Proper metabolic activities facilitate T cell expansion and antitumor function; however, the mechanisms underlying disruption of the T cell metabolic program and function in the tumor microenvironment (TME) remain elusive. Here, we show a zinc finger protein 91-governed (ZFP91-governed) mechanism that disrupts the metabolic pathway and antitumor activity of tumor-infiltrating T cells. Single-cell RNA-Seq revealed that impairments in T cell proliferation and activation correlated with ZFP91 in tissue samples from patients with colorectal cancer. T cell-specific deletion of Zfp91 in mice led to enhanced T cell proliferation and potentiated T cell antitumor function. Loss of ZFP91 increased mammalian target of rapamycin complex 1 (mTORC1) activity to drive T cell glycolysis. Mechanistically, T cell antigen receptor-dependent (TCR-dependent) ZFP91 cytosolic translocation promoted protein phosphatase 2A (PP2A) complex assembly, thereby restricting mTORC1-mediated metabolic reprogramming. Our results demonstrate that ZFP91 perturbs T cell metabolic and functional states in the TME and suggest that targeting ZFP91 may improve the efficacy of cancer immunotherapy.
\end{abstract}

\section{Introduction}

Tumor-infiltrating T cells play a major role in shaping antitumor immune responses, but these cells often exhibit a progressive loss of effector function in tumors $(1,2)$. Metabolic activity is critical for cell growth and proliferation and intimately linked to $\mathrm{T}$ cell fate and function $(3,4)$. Quiescent $\mathrm{T}$ cells rely on oxidative phosphorylation (OXPHOS) as a primary source of energy, whereas activated $\mathrm{T}$ cells shift to glycolytic metabolism to ensure the generation of sufficient biomass and energy (4). Proper metabolic activities facilitate $T$ cell expansion and effector function, and alterations in metabolic programs result in $\mathrm{T}$ cell functional disorders (5-7). Metabolic fitness in T cells is usually modified by the tumor microenvironment (TME) leading to $\mathrm{T}$ cell dysfunction $(8,9)$; however, the associated mechanisms are incompletely understood.

Signaling pathways are responsible for $\mathrm{T}$ cell metabolic reprogramming and antitumor activity in the $\operatorname{TME}(10,11)$. The serine/ threonine protein phosphatase 2A (PP2A) has been implicated in the regulation of $\mathrm{mTOR}$ signaling to sustain the metabolic programs and effector function of T cells $(12,13)$. Elevated PP2A activity in Tregs maintains a metabolic profile essential for Treg-suppressive function $(12,14)$. Potassium-mediated suppression of $\mathrm{T}$ cell function in tumors is dependent on PP2A phosphatase activity (13). Silencing of Ppp2r2d, a regulatory subunit of PP2A, enhances the cytotoxic function of tumor-infiltrating lymphocytes (TILs)

Conflict of interest: The authors have declared that no conflict of interest exists. Copyright: () 2021, American Society for Clinical Investigation.

Submitted: September 15, 2020; Accepted: August 11, 2021; Published: October 1, 2021. Reference information: J Clin Invest. 2021;131(19):e144318.

https://doi.org/10.1172/JCl144318.
$(15,16)$. Pharmacological inhibition of PP2A synergizes with immune checkpoint blockade to strengthen antitumor immune responses in the treatment of solid tumors (17). These findings suggest that PP2A acts as a negative regulator of antitumor $\mathrm{T}$ cell immunity. It is well known that PP2A is the assembly product of 3 distinct subunits, the structural A (PP2Aa), regulatory B (PP2Ab), and catalytic C (PP2Ac) subunits (18). Nevertheless, the underlying mechanisms that orchestrate the holoenzyme assembly of PP2A to disrupt the T cell metabolic pathway and antitumor functions remain unclear.

Zinc finger protein 91 (ZFP91) as an E3 ubiquitin (Ub) ligase is a potentially important oncogenic protein in multiple tumor types, and its oncogenic activities include the suppression of tumor apoptosis, the promotion of tumor growth, and the acceleration of tumor metastasis (19-21). The physiological function of ZFP91 in effector T cell activity in the TME is still unclarified. In this study, our single-cell RNA-Seq (scRNA-Seq) analysis of tumor-infiltrating $\mathrm{T}$ cells revealed that impairments in $\mathrm{T}$ cell proliferation and activation correlated with ZFP91 in tissue samples from patients with colorectal cancer (CRC). Using a mouse strain with a conditional targeted deletion of $Z f p 91$ in T cells, we found that ZFP91 dampened T cell antitumor function in vivo and inhibited $\mathrm{T}$ cell activation and proliferation in vitro. In consequence, targeting ZFP91 in T cells synergized with immunotherapy to delay tumor growth. Mechanistically, T cell antigen receptorinduced (TCR-induced) ZFP91 cytosolic translocation facilitated ZFP91-mediated PP2Ac ubiquitination and PP2A holoenzyme assembly, thereby inhibiting mTORC1-mediated T cell glycolytic metabolism and antitumor function. Our results demonstrate that ZFP91 induced PP2A complex assembly to repress T cell metabolic and functional fitness in the TME. These findings sug- 
A

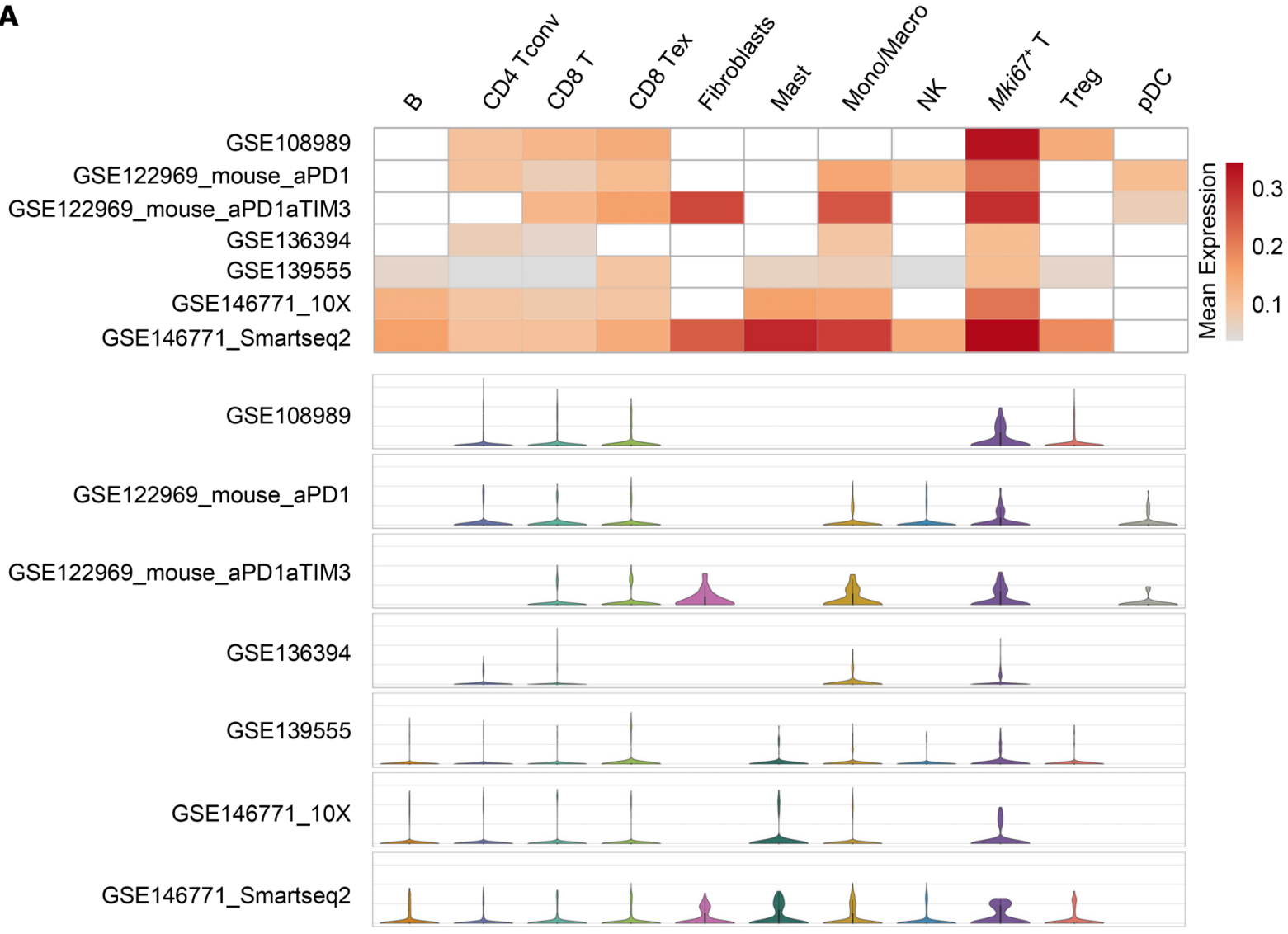

B

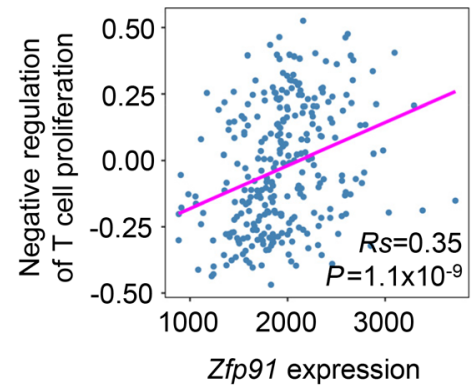

c

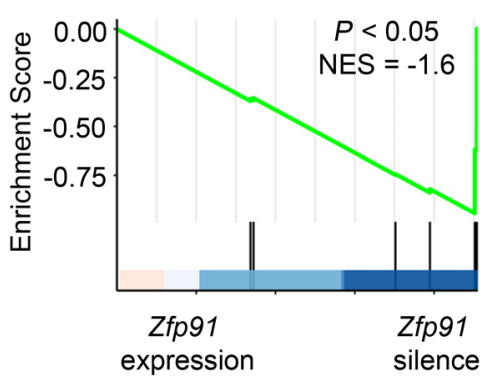

D

T cell activation
0.3

\section{E $O$ Zfp91 $1^{\text {high }}$ T cells $\bullet Z f p 91^{\text {low }}$ T cells}

F $\quad$ Zfp91 $1^{\text {high }}$ T cells $\bullet$ Zfp91low $T$ cells
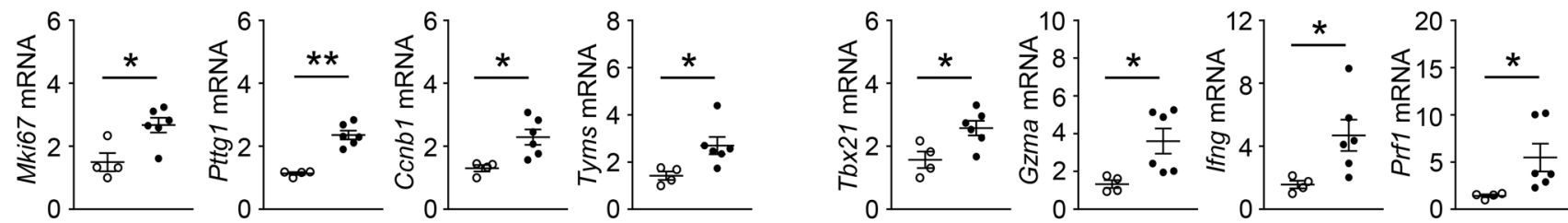

Figure 1. Impairments in T cell proliferation and activation are correlated with ZFP91 in CRC. (A) The heatmap shows the average mRNA expression of ZFP91, and the violin plot shows the distribution of ZFP91 mRNA expression in different cell types from 7 scRNA-Seq data sets for COAD. B, B cells; CD4 Tconv, CD4+ conventional T cells; CD8 T, CD8+ T cells; CD8 Tex, exhausted CD8+ T cells; Mast, mast cells; Mono/Macro, monocytes and macrophages; NK, natural killer cells; Mki67 $7^{+}$, proliferating Mki67 ${ }^{+}$T cells; pDC, plasmacytoid DCs. (B) Spearman's correlation of mRNA expression of Zfpg1 and the GSVA score for negative regulation of T cell proliferation in TCGA COAD database. (C and D) GSEA of the signature genes for the regulation of $C D 8^{+} \alpha \beta$ T cell proliferation (C) and $\alpha \beta$ T cell activation (D) in ZFP91-expressing and ZFP91-silenced T cells. NES, normalization enrichment score. (E and $\mathbf{F}$ ) qRT-PCR analysis of genes associated with T cell proliferation (E) and activation (F) in tumor-infiltrating T cells from CRC. The normalized Zfp91 expression value of tumor-infiltrating T cells with the lowest expression of Zfp91 was set at 1 . The normalized Zfpg1 expression values of Zfpg1 hi T cells were higher than $2(n=4)$, and those of $Z f p 91^{10} \mathrm{~T}$ cells were less than $2(n=6)$. Data in $\mathbf{E}$ and $\mathbf{F}$ are representative of 3 independent experiments. Data are represented as the mean $\pm \mathrm{SEM}$. ${ }^{*} P<0.05$ and ${ }^{* *} P<0.01$ by calculated by permutation test ( $\mathbf{C}$ and $\mathbf{D})$ and 2-tailed Student's $t$ test (E and $\mathbf{F})$. 


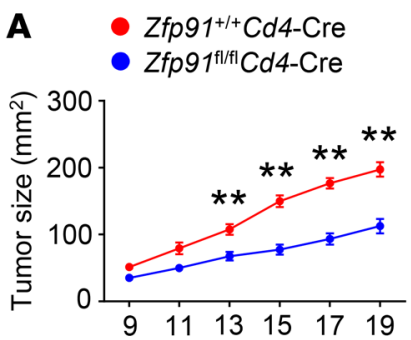

Days after tumor injection

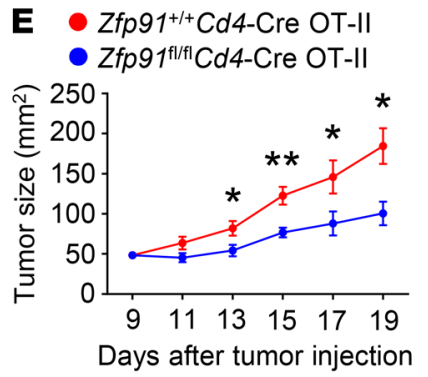

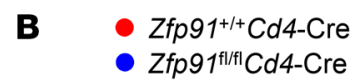

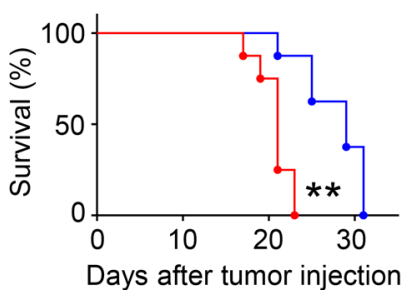

F $\quad$ Zfp91 ${ }^{+/+} \mathrm{Cd}$ 4-Cre OT-II

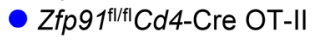

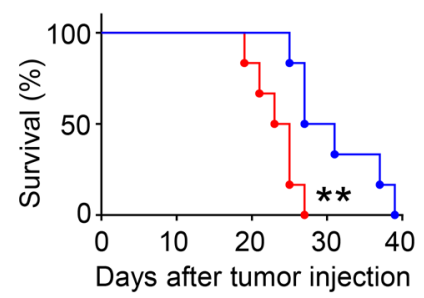

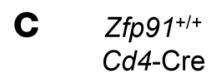

Zfp9 $1^{|f| f \mid}$
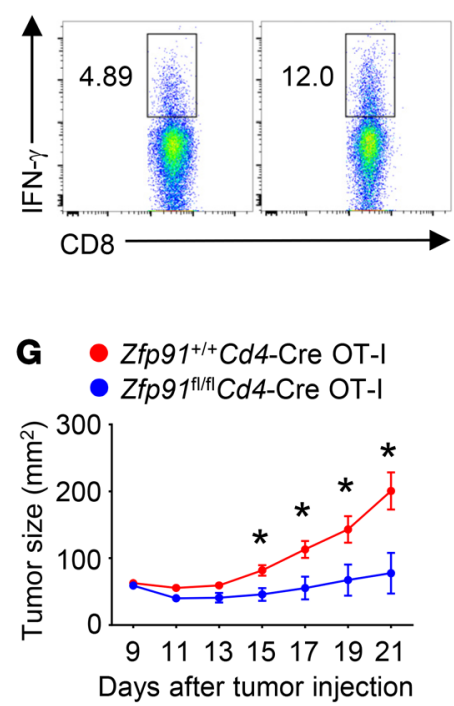

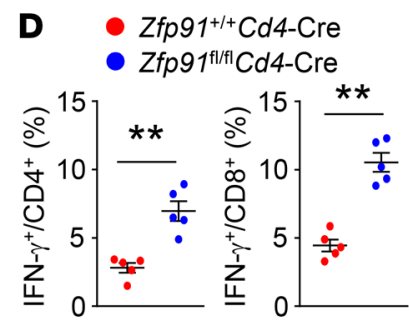

H Zfp91 9 1/+C Cd4-Cre OT-I - Zfp91 ${ }^{\mathrm{Il|f|} C} \mathrm{Cd} 4-\mathrm{Cre}$ OT-I

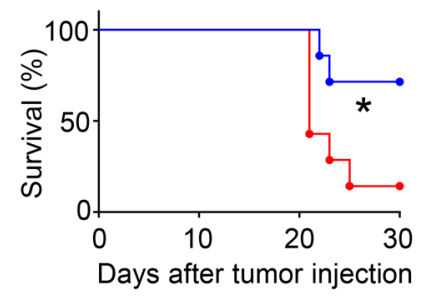

Figure 2. ZFP91 dampens T cell antitumor functions. (A and B) Tumor progression (A) and survival curves (B) for $Z f p 91^{+/+} C d 4-C r e$ and $Z f p g 1^{f / / f l} C d 4-C r e$ mice that received a s.c. injection of $5 \times 10^{5} \mathrm{MC} 38$ murine colon cancer cells $(n=8)$. (C and $\left.\mathbf{D}\right)$ Flow cytometric analysis of T cells in tumors of $Z f p 91^{+/+}$ Cd4-Cre and Zfp9 $f^{f / f f l}$ Cd4-Cre mice injected s.c. with MC38 murine colon cancer cells (day 14). The data are presented as representative plots (C) and as summary graphs (D) $(n=5)$. (E and $\mathbf{F})$ Tumor progression $(\mathbf{E})$ and survival curves (F) for B6.SJL mice that received an i.v. injection of $2 \times 10^{6} \mathrm{Zfp} 91^{+/+} \mathrm{Cd4}-\mathrm{Cre}$

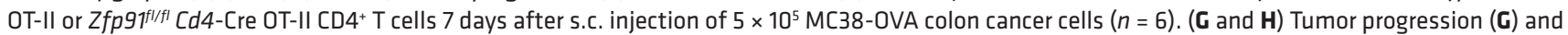

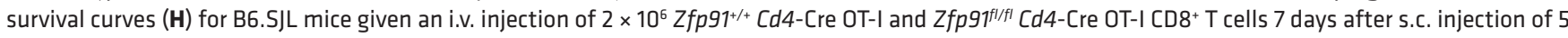
$\times 10^{5}$ MC38-OVA colon cancer cells $(n=7)$. Experiments were independently repeated 3 times. Data are presented as the mean $\pm S E M$. ${ }^{*} P<0.05$ and ${ }^{* *} P<$ 0.01 , by 2-tailed Student's $t$ test (A, D, E, and $\mathbf{G}$ ) and log-rank (Mantel-Cox) test (B, F, and $\mathbf{H})$.

gest that targeting ZFP91 holds great promise to unleash the full antitumor activity of tumor-infiltrating T cells and could help in the design of innovative strategies to improve the efficacy of cancer immunotherapy.

\section{Results}

Impairments in $T$ cell proliferation and activation correlate with ZFP91 in CRC. To investigate the potential function of ZFP91 in $\mathrm{T}$ cell activity in the TME, we analyzed the mRNA expression profile of ZFP91 in different cell types from colon adenocarcinoma (COAD) tissue using the scRNA-Seq database in the Tumor Immune Single-Cell Hub (TISCH) (22-26). Compared with other cell types, proliferating $M k i 67^{+} \mathrm{T}$ cells exhibited relatively high mRNA expression levels of ZFP91 in 7 scRNA-Seq human and mouse COAD data sets (Figure 1A), indicating that ZFP91 may participate in the regulation of $\mathrm{T}$ cell proliferation in the TME. To further explore the role of ZFP91 in tumor-infiltrating T cells, we analyzed the correlation between $\mathrm{T}$ cell proliferation and ZFP91 mRNA expression in The Cancer Genome Atlas (TCGA) COAD data set (27). Interestingly, we observed a significantly positive correlation between the gene set variation analysis (GSVA) score for negative regulation of $\mathrm{T}$ cell proliferation and ZFP91 expression in patients with CRC (Figure 1B). Using previously published scRNA-Seq data for human colorectal TILs (22), we found that ZFP91-silenced CD8 ${ }^{+} \mathrm{T}$ cells exhibited enrichment in $\mathrm{T}$ cell proliferation-related genes (Figure 1C). Furthermore, the expression of $\mathrm{T}$ cell activation-associated genes was also upregulated in ZFP91-silenced T cells from CRC tissue (Figure 1D). Subsequent- ly, we verified these findings using CRC tissue samples. Indeed, we found that tumor-infiltrating T cells with low ZFP91 expression contained abundant transcription of genes associated with $\mathrm{T}$ cell proliferation and activation (Figure 1, E and F). These data suggest that impairments in $\mathrm{T}$ cell proliferation and activation correlate with ZFP91 in CRC.

ZFP91 dampens $T$ cell antitumor functions. To verify the physiological importance of ZFP91 in T cell antitumor activity, we crossed $Z f p 91$-floxed mice with $C d 4$-Cre mice to generate $Z f p 91$ T cell-conditional KO mice $\left(Z f p 91^{f l / f l} C d 4\right.$-Cre), in which ZFP91 was specifically deleted in T cells (Supplemental Figure 1A; supplemental material available online with this article; https:/doi. org/10.1172/JCI144318DS1). T cell-specific deletion of Zfp91 did not affect $\mathrm{T}$ cell development or peripheral $\mathrm{T}$ cell homeostasis in 6-week-old mice (Supplemental Figure 1, B and C). In addition, the Treg cell percentages in the thymus, spleen, and peripheral lymph nodes of 6-week-old $Z f p 91^{+/+} C d 4$-Cre mice and $Z f p 91^{f l / f l} C d 4$-Cre mice were comparable (Supplemental Figure 1D). Using an MC38 COAD model, we examined the role of ZFP91 in regulating antitumor immune responses. Compared with WT mice, $Z f p 91^{f l / f l} C d 4$-Cre mice had profound reductions in MC38 tumor size and tumor-induced lethality (Figure 2, A and B). In addition, MC38 cell-challenged Zfp91 $91^{f / f l} C d 4$-Cre mice had an increased frequency of IFN- $\gamma$-producing $\mathrm{CD} 4^{+}$and $\mathrm{CD}^{+}$effector $\mathrm{T}$ cell infiltration into the tumors (Figure 2, C and D). Parallel studies revealed that the $Z f p 91^{f l / f l} \mathrm{Cd} 4$-Cre mice also displayed enhanced antitumor immunity in a B16 melanoma model (Supplemental Figure 2, A-C). 
A

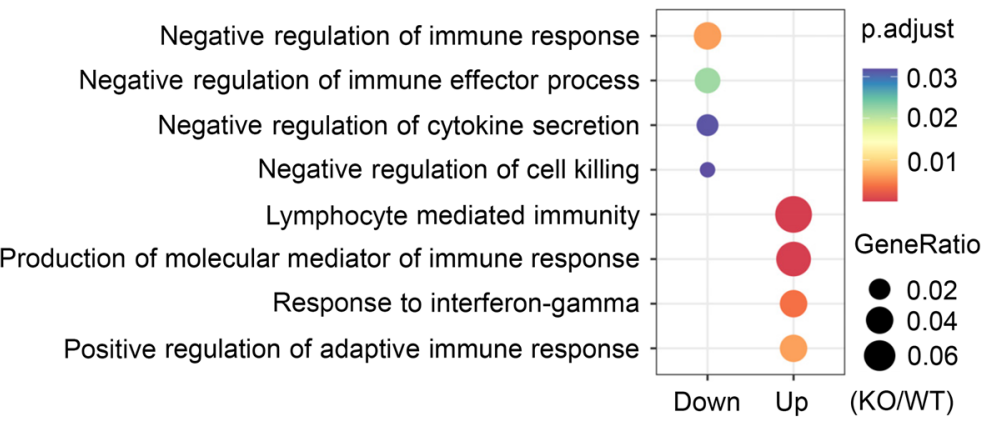

B

Negative regulation of immune respons
Negative regulation of immune effector process
Negative regulation of cytokine productio
Negative regulation of $\mathrm{T}$ cell mediated immunity
IFN- $\gamma$-mediated signaling pathwa
Regulation of response to cytokine stimulus
Regulation of response to IFN

Positive regulation of cytokine-mediated signaling pathway

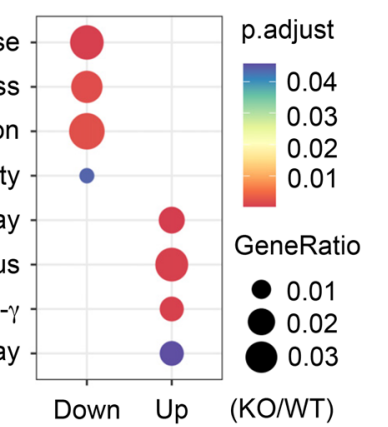

$\mathbf{E}$

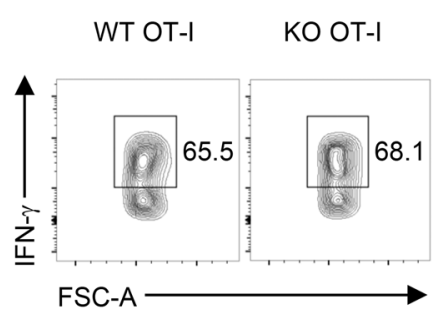

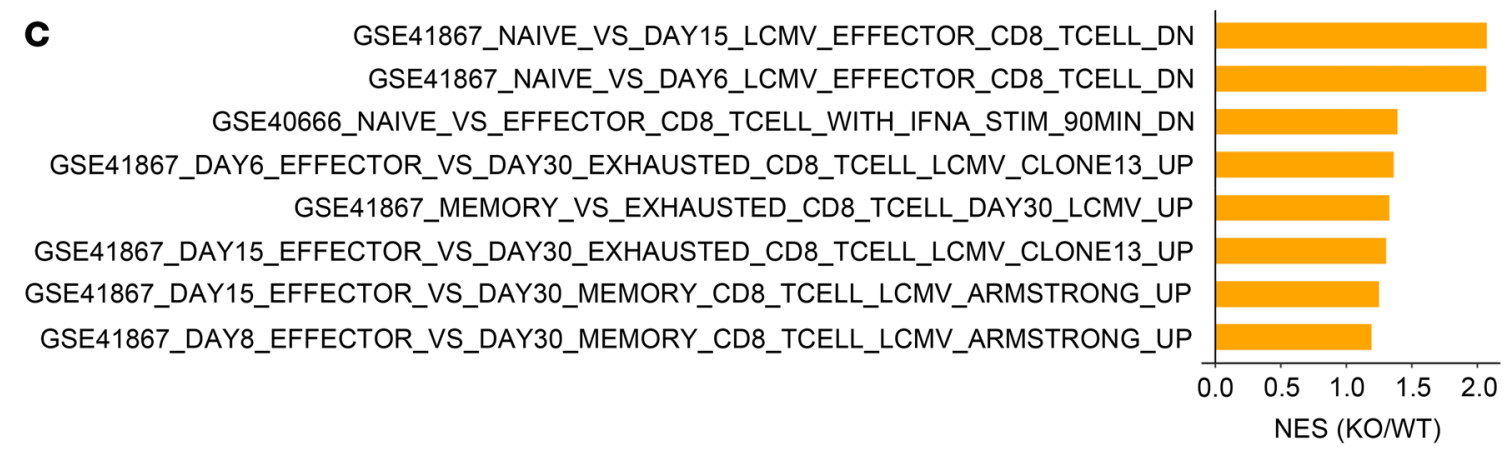

Figure 3. ZFP91 disturbs antitumor activity of tumor-infiltrating T cells. (A and B) Gene ontology enrichment analysis of upregulated (Up) and down-

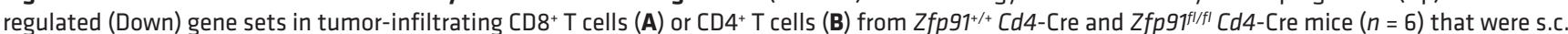
injected with MC38 murine colon cancer cells (day 14). p.adjust, adjusted $P$ value. (C) GSEA enrichment plots of the indicated signatures in tumor-infil-

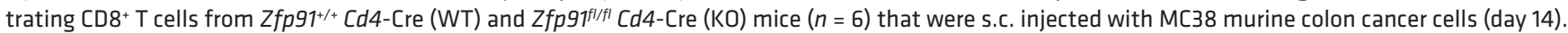
(D) Number of OT-I cells in tumor tissues from MC38-OVA tumor-bearing mice with transferred CFSE-labeled WT OT-I and CTV-labeled KO OT-I cells (on day 7 after adoptive transfer of OT-I cells) $(n=4)$. (E-C) Percentage and number of IFN- $\gamma^{+}$OT-I cells (E and $\left.\mathbf{F}\right)$ or Ki-67+ OT-I cells (C) in tumor tissues from MC38-OVA tumor-bearing mice with transferred OT-I cells (on day 7 after adoptive transfer of OT-I cells) $(n=4)$. Experiments were independently repeated 4 times $(\mathbf{D}-\mathbf{G})$. Data are presented as the mean \pm SEM. ${ }^{* *} P<0.01$, by 2 -tailed Student's $t$ test.

To confirm the role of ZFP91 in T cell antitumor function, we adoptively transferred $\mathrm{CD} 4^{+}$or $\mathrm{CD} 8^{+} \mathrm{T}$ cells isolated from $Z f \mathrm{fp} 9 \mathrm{f}^{\mathrm{fl} / \mathrm{l}}$ Cd4-Cre OVA-specific TCR-transgenic (OT-II or OT-I, respectively) mice into MC38-OVA tumor-bearing WT mice. As expected, the mice transferred with $Z f p 91^{f / f l} \mathrm{Cd}$-Cre OT-II T cells showed more suppressed tumor growth and prolonged survival (Figure 2, $\mathrm{E}$ and F). Consistently, the mice that received $Z f p 91^{f / / l} \mathrm{Cd}$-Cre OT-I T cells showed much greater efficiency in controlling tumor growth and tumor-induced lethality (Figure 2, G and H). Collectively, these results suggest that ZFP91 in T cells perturbs antitumor immune responses.

ZFP91 disturbs the antitumor activity of tumor-infiltrating $T$ cells. To demonstrate that ZFP91 disturbs $\mathrm{T}$ cell antitumor activity in the TME, we isolated tumor-infiltrating $\mathrm{T}$ cells from tumor-bearing $Z f p 91^{+/+} C d 4$-Cre mice and $Z f p 91^{f / f / l} C d 4$-Cre mice for the transcriptomic analysis. We noticed that negative regulation of the immune effector process was downregulated in both $\mathrm{CD}^{+}$and $\mathrm{CD}^{+} \mathrm{T}$ cells from the tumors of $Z \mathrm{fp}^{\mathrm{g}} 91^{\mathrm{fl} / \mathrm{l}} \mathrm{Cd}$-Cre mice (Figure 3, A and B). By contrast, positive regulation of adaptive immune responses was upregulated in tumor-infiltrating ZFP91-deficient CD8 ${ }^{+} \mathrm{T}$ cells (Figure 3A). Gene set enrichment analysis (GSEA) also confirmed that effector $\mathrm{T}$ cell signatures were enriched in tumor-infiltrating ZFP91-deficient $\mathrm{CD} 8^{+} \mathrm{T}$ cells (Figure 3C). To better define the phenotypes of tumor-infiltrating ZFP91-deficient T cells, we adoptively transferred CFSE-labeled WT OT-I cells and CTV-labeled ZFP91-deficient OT-I cells into the same tumor-bearing mice. Notably, we observed increased intratumoral infiltration of ZFP91-deficient $\mathrm{CD} 8^{+} \mathrm{T}$ cells in the tumor-bearing mice that were transferred with WT and ZFP91-deficient OT-I cells (Figure 3D). Importantly, IFN- $\gamma$-producing 


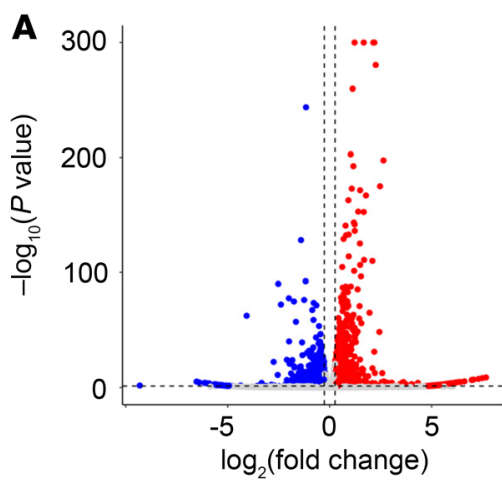

B

Fatty acid metabolic process

Cellular amino acid metabolic process

Positive regulation of cytokine production

IFN- $\gamma$ production

Positive regulation of $\mathrm{T}$ cell activation

Positive regulation of $\mathrm{T}$ cell proliferation

T cell-mediated cytotoxicity

Regulation of glycolytic process

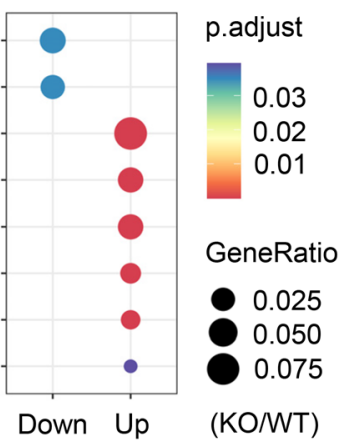

C

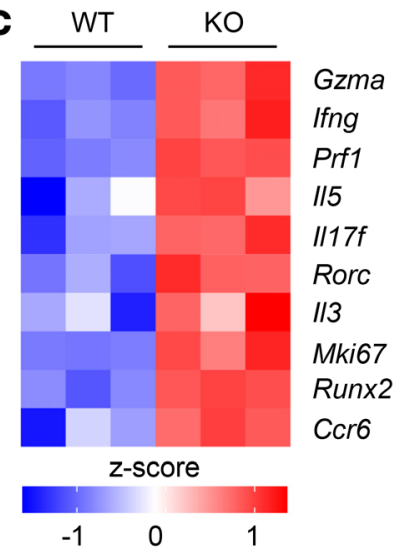

E $\mathrm{CD}^{+} \mathrm{T}$ cells

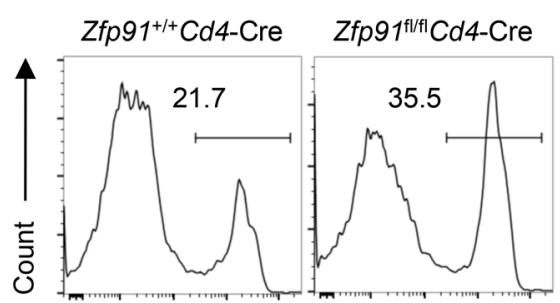

$\mathrm{Ki}-67$

$\mathbf{F}$

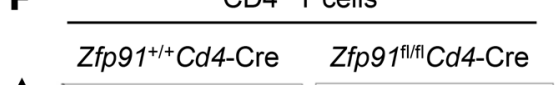

D $\quad \mathrm{Zfp} 91^{+++} \mathrm{Cd} 4-\mathrm{Cre}$

- Zfp9 $1^{\text {fllirl } C d 4-C r e ~}$
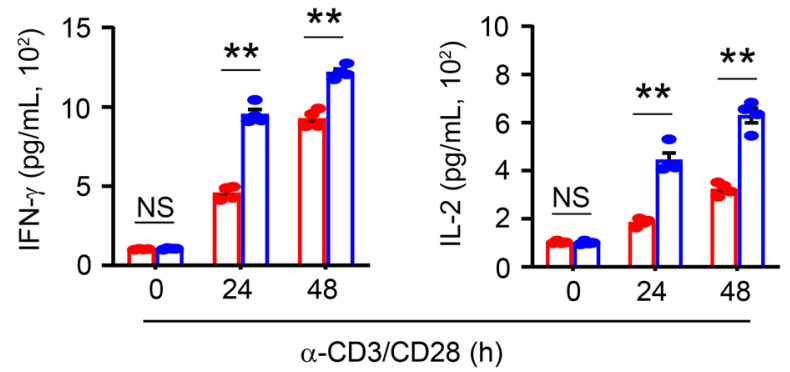

$\alpha-C D 3 / C D 28(h)$
$\mathrm{CD}^{+} \mathrm{T}$ cells

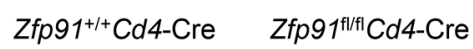
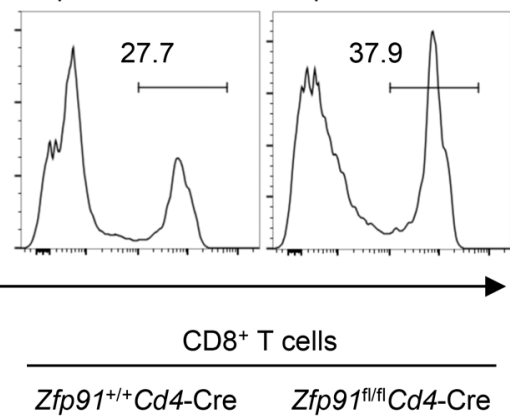

Zfp91 ${ }^{+/+} \mathrm{Cd} 4-\mathrm{Cre} \quad$ Zfp91 ${ }^{\mathrm{fl} / \mathrm{l}} \mathrm{C}$ C4-Cre

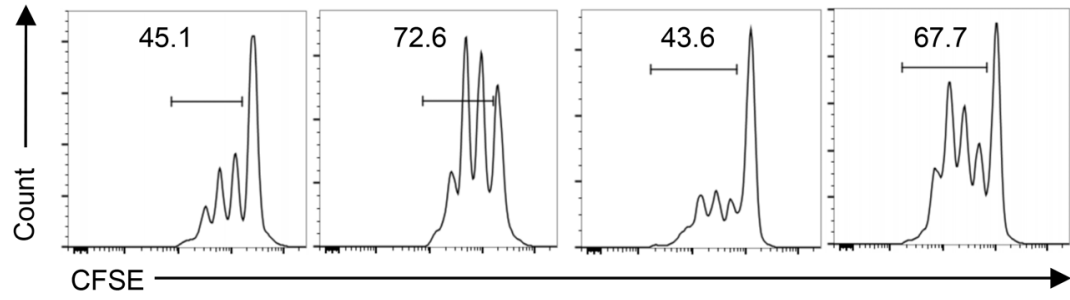

- Zfp91 $91^{+++} \mathrm{Cd} 4-\mathrm{Cre}$

- Zfp91 ${ }^{\mathrm{fl} / \mathrm{fl}} \mathrm{Cd} 4 \mathrm{-Cre}$

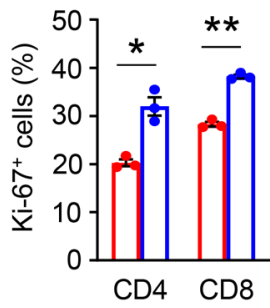

- Zfp91 ${ }^{+/+}$Cd4-Cre

- Zfp91/l/fl Cd4-Cre

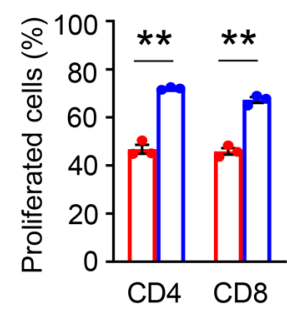

Figure 4. ZFP91 deficiency promotes $\mathrm{T}$ cell activation and proliferation. (A) Volcano plot comparing global gene expression profiles between $Z f p 91^{+/+}$ Cd4-Cre (WT) and Zfpg1 $1^{f / f l} \mathrm{Cd} 4$-Cre (KO) CD90.2+ T cells stimulated with anti-CD3 and anti-CD28 antibodies for 24 hours. The red dots represented the transcripts with increasing expression values, whereas the blue dots signify transcripts with decreasing expression values in $Z f p 91^{f l / f l} \mathrm{Cd4}-\mathrm{Cre} C D 90.2^{+} \mathrm{T}$

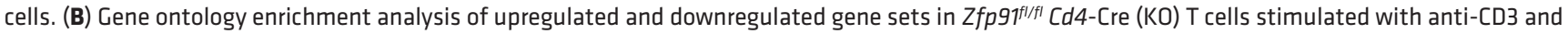
anti-CD28 antibodies for 24 hours. (C) Heatmap of upregulated genes associated with T cell activation and proliferation in ZFP91-deficient T cells relative

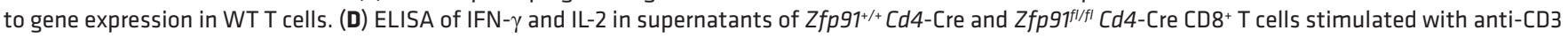

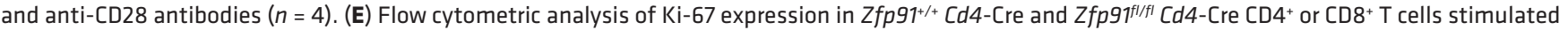

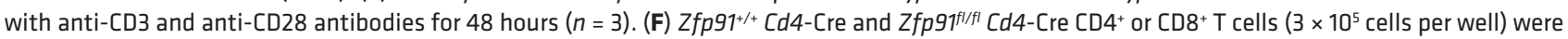
labeled with CFSE and cultured with irradiated splenocytes depleted of T cells $\left(1 \times 10^{5}\right.$ cells per well) in the presence of anti-CD3 and anti-CD28 antibodies for 72 hours for T cell proliferation assays $(n=3)$. Experiments were independently repeated 3 times. Data are presented as the mean \pm SEM. ${ }^{*} P<0.05$ and ${ }^{* *} P<0.01$, by 2 -tailed Student's $t$ test. 

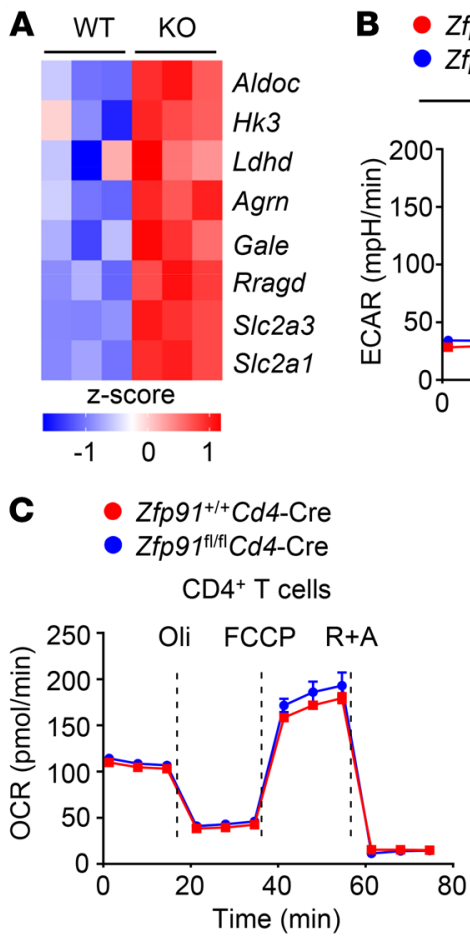

$\mathbf{F}$

\section{- WT OT-I}

- KO OT-I

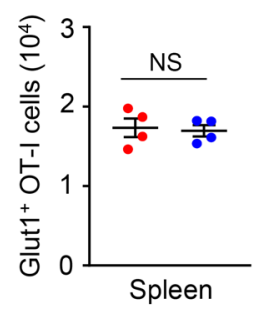

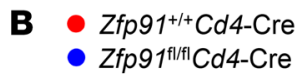
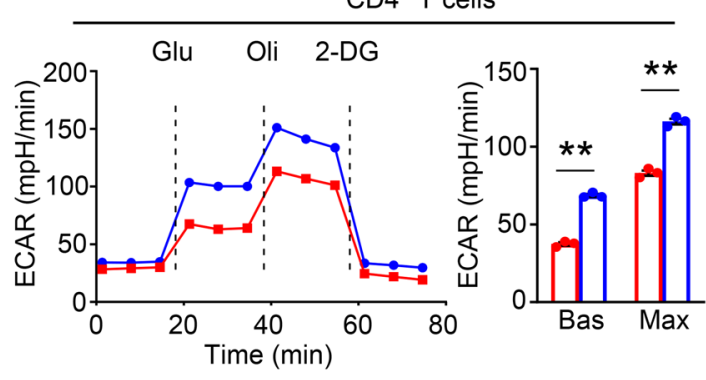

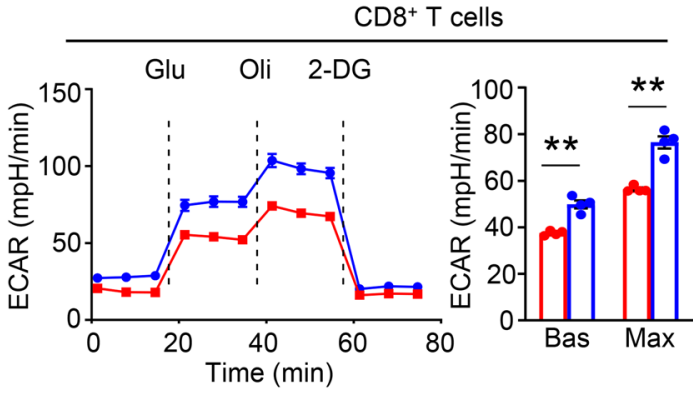

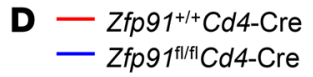

E - $z f p 91^{1++} \mathrm{Cd} 4-\mathrm{Cre}$

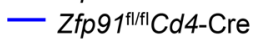
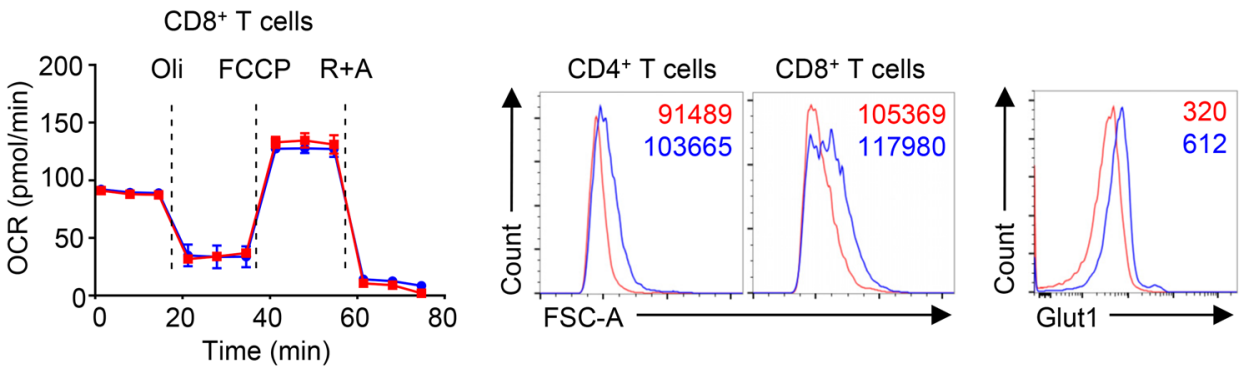

G
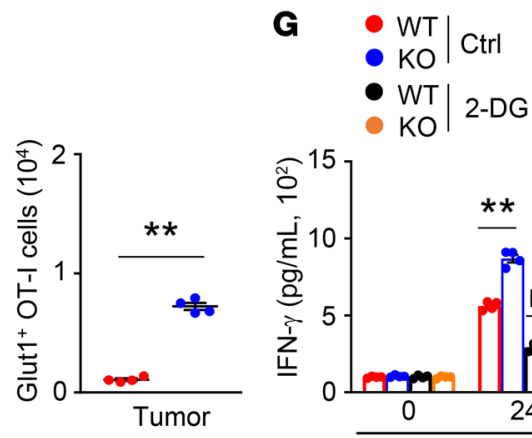

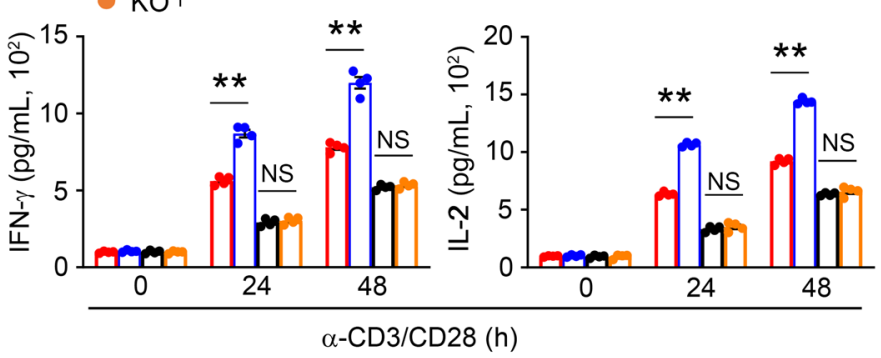

H
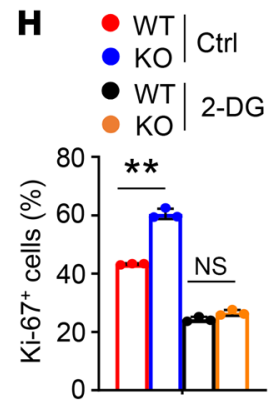

Figure 5. ZFP91 suppresses T cell glycolytic metabolism. (A) Heatmap of upregulated genes associated with T cell glycolysis in ZFP91-deficient T cells relative

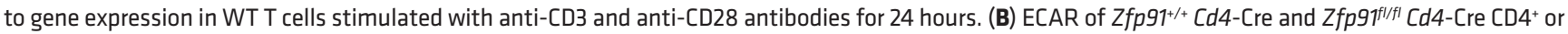
CD8 ${ }^{+}$T cells stimulated with anti-CD3 and anti-CD28 antibodies for 24 hours, at baseline (Bas) and in response to glucose (Clu), oligomycin (Oli), and 2-DC ( $n=$ 3 or 4). Max, maximum. (C) OCRs of $Z f p 91^{+/+} \mathrm{Cd} 4-\mathrm{Cre}$ and $\mathrm{Zfp} 91^{f / / f l} \mathrm{Cd} 4-\mathrm{Cre} C \mathrm{CD} 4^{+}$or CD8 ${ }^{+} \mathrm{T}$ cells stimulated with anti-CD3 and anti-CD28 antibodies for 24 hours, at baseline and in response to oligomycin, FCCP, and rotenone plus antimycin $(R+A)(n=3-4)$. (D) Flow cytometric analysis of cell size (forward scatter area

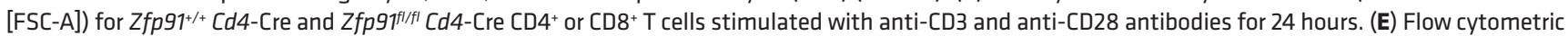
analysis of Clut1 expression in T cells in tumors from Zfpg1+/+ Cd4-Cre and Zfp91//fl Cd4-Cre mice injected s.c. with MC38 murine colon cancer cells (day 14). (F) Number of Glut1 $1^{+}$OT-I cells in the spleen and tumor from MC38-OVA tumor-bearing mice transferred with OT-I cells (on day 7 after adoptive transfer of OT-I cells) $(n=4)$. ( $(\mathbf{G}$ and $\mathbf{H})$ ELISA of IFN- $\gamma$ and IL-2 in supernatants $(\mathbf{G}, n=4)$ and flow cytometric analysis of Ki-67 expression ( $\mathbf{H}, n=3)$ in Zfp91+/+ Cd4-Cre (WT) and $\mathrm{Zfp} 97^{\mathrm{fl} / \mathrm{fl}} \mathrm{Cd} 4$-Cre (KO) T cells stimulated with anti-CD3 and anti-CD28 ( $\alpha$-CD3/CD28) antibodies for 48 hours in the presence of 2-DC (2 mM) or control (Ctrl). Experiments were independently repeated 3 times. Data are presented as the mean \pm SEM. ${ }^{*} P<0.01$, by 2-tailed Student's $t$ test.

ZFP91-deficient OT-I cells showed dramatic accumulation in the TME, although the frequency of IFN- $\gamma$-producing WT OT-I cells and ZFP91-deficient OT-I cells was similar (Figure 3, E and F). Moreover, ZFP91-deficient OT-I cells expressed higher levels of Ki-67 than did WT OT-I cells in the TME (Figure 3G). By contrast, splenic WT OT-I cells and ZFP91-deficient OT-I cells displayed comparable levels of IFN- $\gamma$ and $\mathrm{Ki}-67$ in the tumor-bearing mice that were transferred with WT and ZFP91-deficient OT-I cells (Supplemental Figure 3, A-D). These data suggest that ZFP91 is indispensable for the functional state of tumor-infiltrating $\mathrm{T}$ cells.
ZFP91 inhibits T cell activation and proliferation. To understand the mechanism by which ZFP91 regulates T cell activity, we performed transcriptomic analysis of $Z f p 91^{+/+} C d 4$-Cre and $Z f p 91^{f / f l}$ Cd4-Cre CD90. $2^{+}$T cells stimulated with anti-CD3 and anti-CD28 antibodies for 24 hours (Figure 4A). Interestingly, the levels of genes involved in $\mathrm{T}$ cell activation and proliferation were upregulated in ZFP91-deficient T cells (Figure 4, B and C). Moreover, ZFP91 deficiency augmented the TCR- and CD28-stimulated production of effector cytokines, such as IFN- $\gamma$ and IL-2, in ZFP91-deficient $\mathrm{CD}^{+} \mathrm{T}$ cells (Figure 4D). In addition, expression of the cell 
A

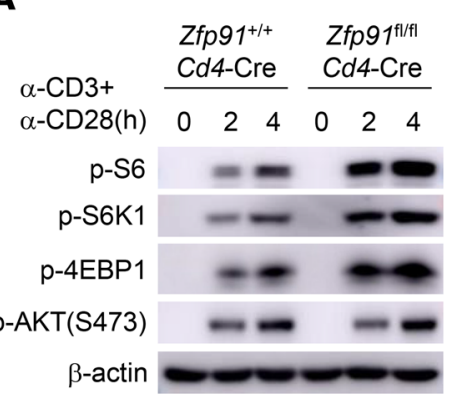

B

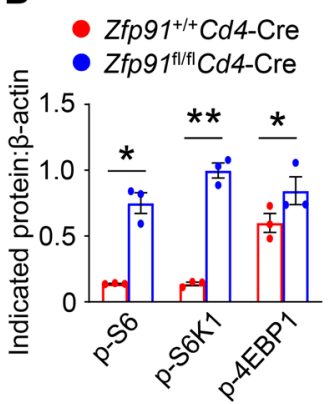

C
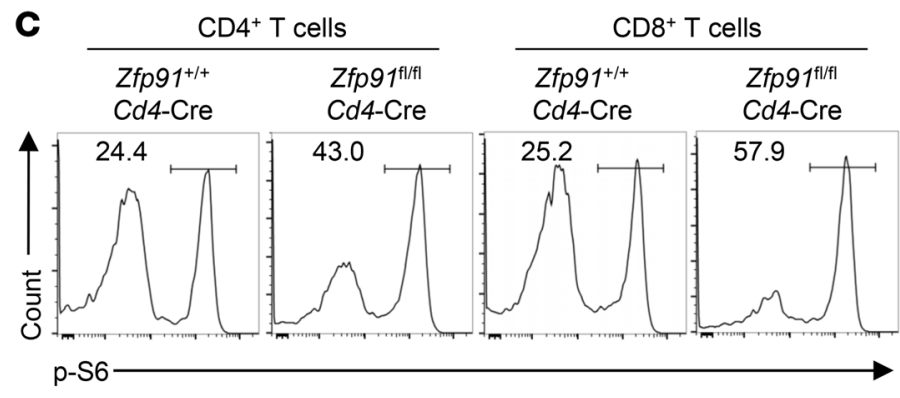

E
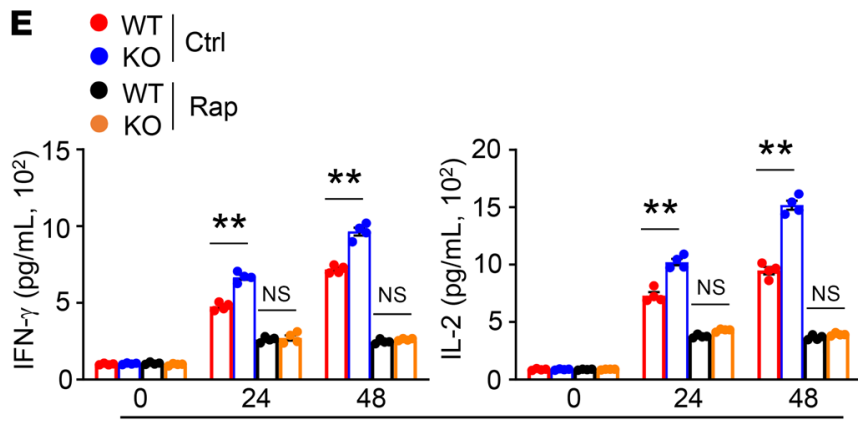

$\alpha-C D 3 / C D 28$ (h)

H

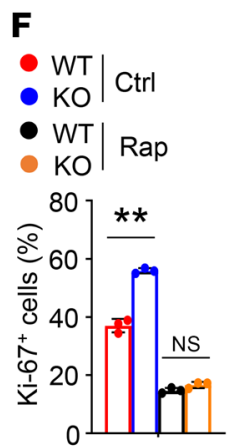

G
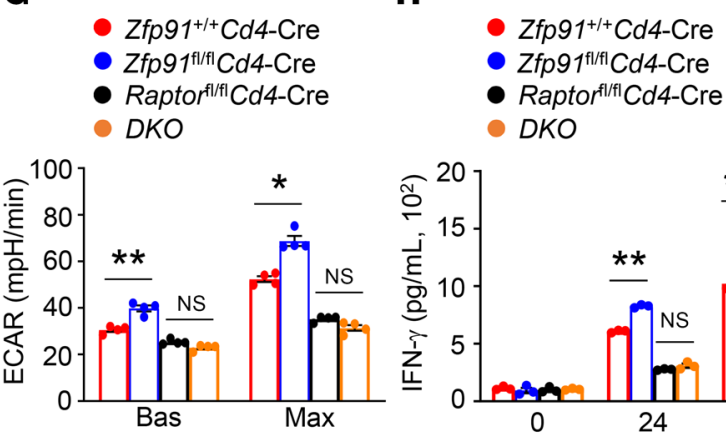

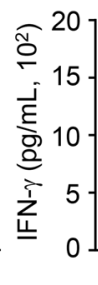

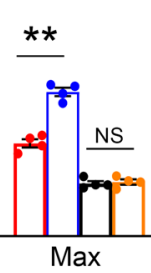

DKO
I

- Zfp91++ Cd4-Cre - Zfp91/l/fil Cd4-Cre - Raptor rililild4-Cre - $D K O$

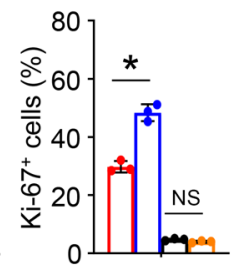

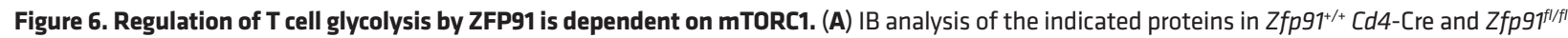

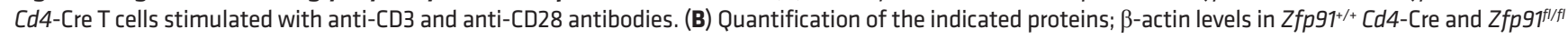

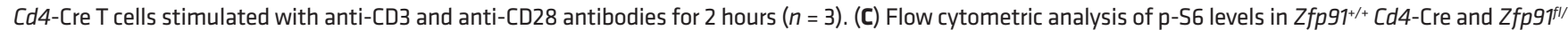
${ }^{f l}$ Cd4-Cre CD4 ${ }^{+}$or CD8 ${ }^{+}$T cells stimulated with anti-CD3 and anti-CD28 antibodies for 2 hours. (D-F) ECAR (D, $\left.n=4\right)$, ELISA of IFN- $\gamma$ and IL-2 in supernatants (E, $n=4)$, and flow cytometric analysis of Ki-67 expression $(\mathbf{F}, n=3)$ in Zfp91+/+Cd4-Cre (WT) and Zfp9 $f^{f / / f l}$ Cd4-Cre (KO) T cells stimulated with anti-CD3 and antiCD28 antibodies for 24 hours (D) or 48 hours (E and F) in the presence of rapamycin (Rap, $1 \mu$ M). (G-I) ECAR (G, $n=4)$, ELISA of IFN- $\gamma$ and IL-2 in supernatants

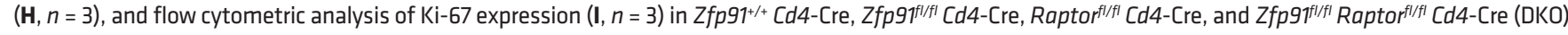
CD90.2 $2^{+}$cells stimulated with anti-CD3 and anti-CD28 antibodies for 24 hours (C) or 48 hours (H and I). Experiments were independently repeated 3 times. Data are presented as the mean \pm SEM. ${ }^{*} P<0.05$ and ${ }^{* *} P<0.01$, by 2 -tailed Student's $t$ test. Max, maximum.

proliferation marker Ki-67 we observed following TCR and CD28 stimulation was increased in ZFP91-deficient $\mathrm{CD}^{+} \mathrm{T}$ cells and $\mathrm{CD}^{+} \mathrm{T}$ cells (Figure 4E). Consistently, we observed enhanced proliferation of ZFP91-deficient CD4 ${ }^{+} \mathrm{T}$ cells and $\mathrm{CD} 8^{+} \mathrm{T}$ cells after TCR and CD28 stimulation (Figure 4F). However, ZFP91-deficient and $\mathrm{WT} \mathrm{CD}^{+} \mathrm{T}$ cells were similar with regard to $\mathrm{T}$ cell differentiation (Supplemental Figure 4, A and B), suggesting that ZFP91 is dispensable for $\mathrm{T}$ cell differentiation. These results indicate crucial roles for ZFP91 in restraining T cell activation and proliferation.

ZFP91 suppresses $T$ cell glycolytic metabolism. Glucose metabolism is required for $\mathrm{T}$ cell activation, proliferation, and effector functions (4). Our transcriptomic analysis revealed that the levels of genes associated with glycolysis, including Slc2a1, Slc2a3, Hk3, and Aldoc, were upregulated in ZFP91-deficient T cells (Figure $5 \mathrm{~A}$ ), which prompted us to examine the involvement of ZFP91 in T cell metabolic programs. Indeed, ZFP91-deficient T cells had significantly higher baseline and maximum glycolytic rates than did ZFP91-sufficient T cells after TCR and CD28 stimulation (Figure 5B), indicating that ZFP91 regulates T cell glycolysis. In contrast, stimulated ZFP91-deficient and ZFP91-sufficient T cells had similar OXPHOS rates, both at baseline and at maximum capacity (Figure 5C), suggesting a dispensable role for ZFP91 in regulating $\mathrm{T}$ cell OXPHOS. Increased aerobic glycolysis supports rapid $\mathrm{T}$ cell growth (28). In line with this, ZFP91-deficient T cells exhib- 
A

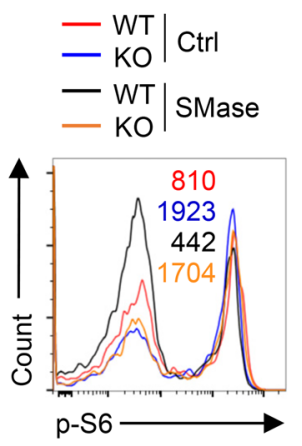

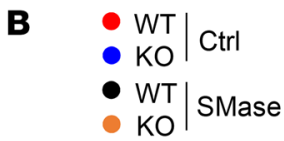
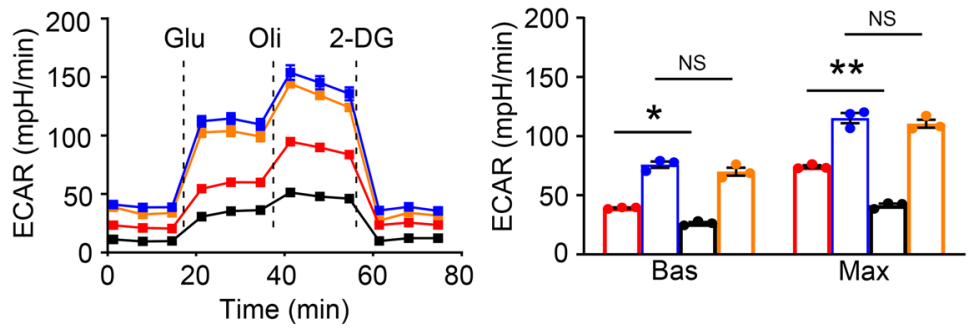

C

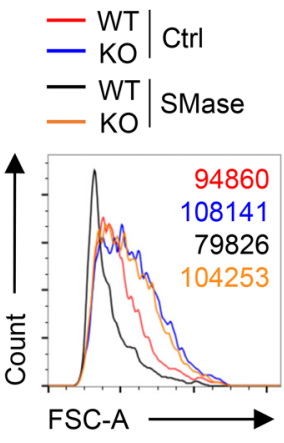

D

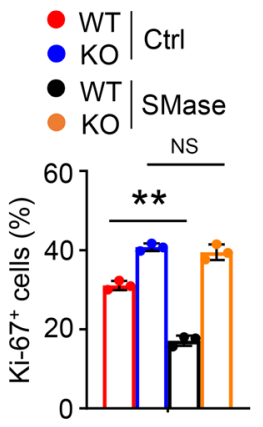

I

EV PP2AC

WT KO WTKO

HA-PP2AC

E
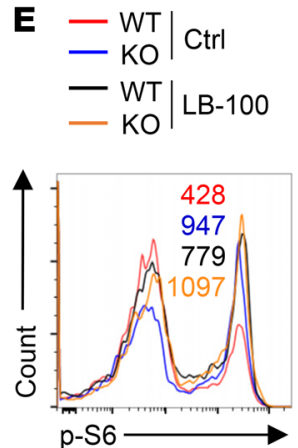

J - ${ }_{\mathrm{KO}} \mid \mathrm{EV}$

- WT ${ }_{\text {KO }} \mid$ PP2AC

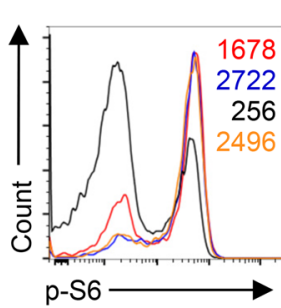

$\mathbf{F}$
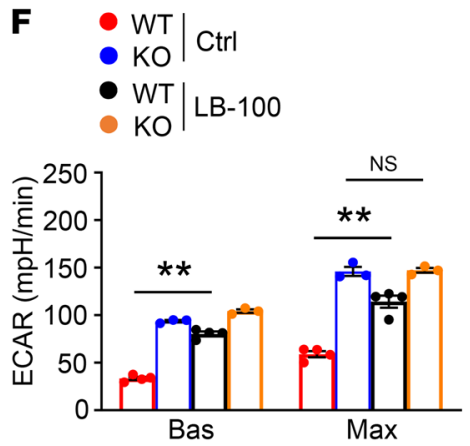

$\mathbf{K}$
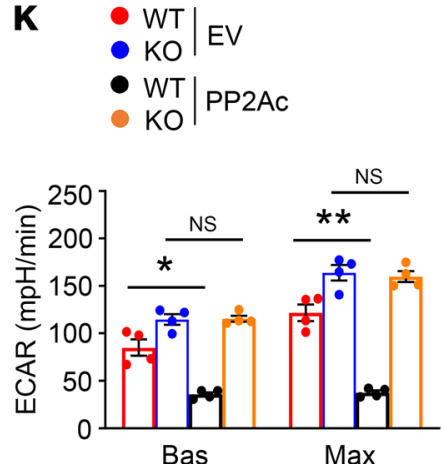

G
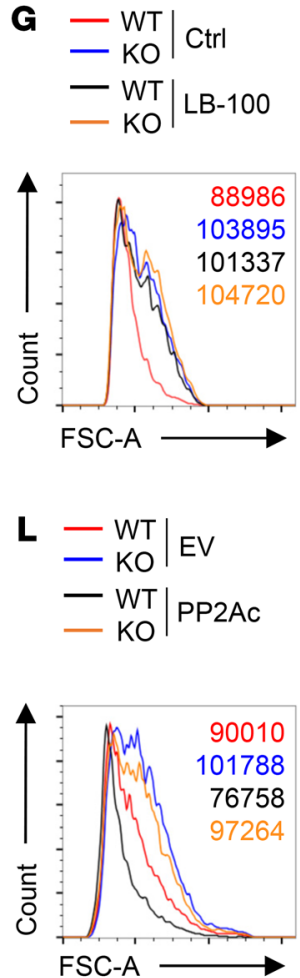

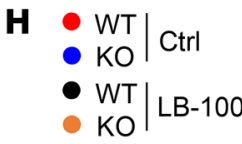

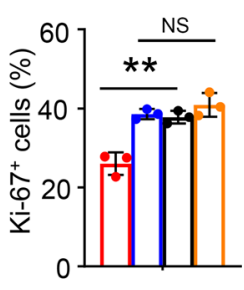

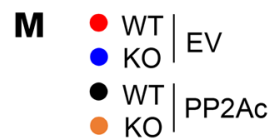

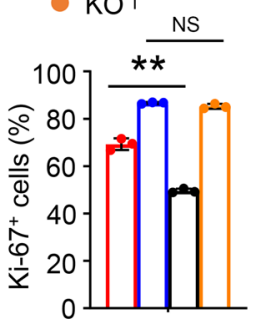

Figure 7. ZFP91 is required for PP2A activity in T cells. (A) Flow cytometric analysis of p-S6 levels in Zfp91+/+ Cd4-Cre (WT) and Zfp91 fl/fl Cd4-Cre (KO) CD90.2+ T cells stimulated with anti-CD3 and anti-CD28 antibodies for 2 hours in the presence of SMase (0.5 U/mL) or control (50\% glycerol in PBS). (B) ECAR of WT and KO T cells stimulated with anti-CD3 and anti-CD28 antibodies for 24 hours in the presence of SMase $(n=3)$ or control. (C and D) Flow cytometric analysis of cell size (C) and Ki-67 expression ( $\mathbf{D}, n=3)$ in WT and KO T cells stimulated with anti-CD3 and anti-CD28 antibodies for 24 hours (for FSC-A detection) or 48 hours (for Ki-67 detection) in the presence of SMase or control. (E) Flow cytometric analysis of p-S6 levels in WT and KO T cells stimulated with anti-CD3 and anti-CD28 antibodies for 2 hours in the presence of LB-100 $(1 \mu \mathrm{M})$ or control. (F) ECAR of WT and KO T cells stimulated with anti-CD3 and anti-CD28 antibodies for 24 hours in the presence of LB-100 $(1 \mu \mathrm{M})$ or control. (G and $\mathbf{H})$ Flow cytometric analysis of cell size (C) and Ki-67 expression ( $\mathbf{H}, n=3)$ in WT and KO T cells stimulated with anti-CD3 and anti-CD28 antibodies for 24 hours (for FSC-A detection) or 48 hours (for Ki-67 detection) in the presence of LB-100 or control. (I) IB analysis of HA-PP2Ac in WT and KO T cells transduced with either an empty vector (EV) or PP2Ac. (J-M) p-S6 expression (J), ECAR (K, $n=4)$, cell size $(\mathbf{L})$, and Ki-67 expression $(\mathbf{M}, n=3)$ for transduced T cells from I stimulated for 2 hours $(\mathrm{J}), 24$ hours (K and $\mathbf{L}$ ), or 48 hours $(\mathbf{M})$ with antibodies against CD3 and CD28. Experiments were independently repeated 3 times. Data are presented as the mean \pm SEM. ${ }^{*} P<0.05$ and ${ }^{* *} P<0.01$, by 2 -tailed Student's $t$ test.

ited increases in cell size (Figure 5D). Consistently, high glucose transporter 1 (Glut1) surface expression, which is associated with increased glycolytic activity, was detectable in tumor-infiltrating ZFP91-deficient T cells (Figure 5E). Moreover, tumor-infiltrating ZFP91-deficient OT-I cells had higher levels of Glut1 compared with WT OT-I cells in the tumor-bearing mice that were transferred WT or ZFP91-deficient OT-I cells (Figure 5F). By contrast, splenic WT OT-I cells and ZFP91-deficient OT-I cells displayed comparable levels of Glut1 in the tumor-bearing mice with transferred OT-I cells (Figure 5F). To define the dependence of effector cytokine production and Ki-67 upregulation on glucose availability, we further activated $\mathrm{T}$ cells in the presence or absence of a glycolysis inhibitor, 2-deoxy-D-glucose (2-DG). After the addition of 2-DG, effector cytokine production and Ki-67 expression were greatly diminished in both WT T cells and ZFP91-deficient T cells (Figure 5, G and H). Importantly, stimulated ZFP91-deficient and 
A

HA-Ub $\frac{\mathrm{Ub}}{++} \frac{\mathrm{K} 48}{++} \frac{\mathrm{K} 63}{++} \frac{\mathrm{K} 63 \mathrm{R}}{++}$

His-ZFP91 -+-+-+-+

Flag-PP2AC ++++++++

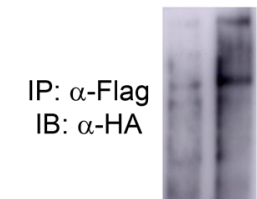

His-ZFP91

Flag-PP2Ac
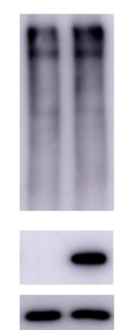
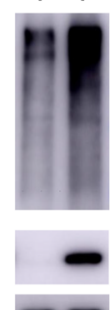

$\infty$
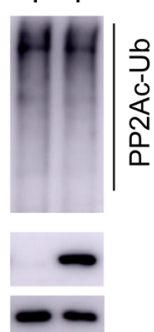

B

IP: $\alpha-P P 2 A c$

IB: $\alpha-U b-K 63$

E $\alpha-C D 3+\frac{\text { WT T cells }}{\alpha-C D 28}$

IP: $\alpha$-ZFP91

IB: $\alpha-P P 2 A C$

Cell lysates

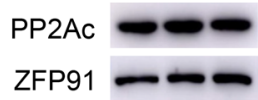

$\mathbf{F}$

\begin{tabular}{|c|c|}
\hline 0 & 4 \\
\hline
\end{tabular}

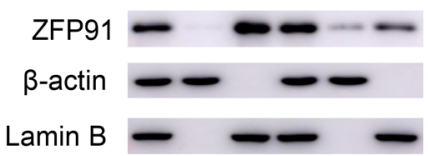

G

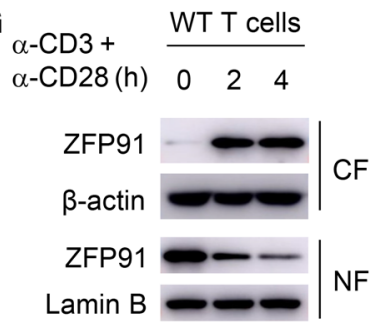

C

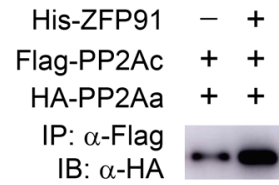

Cell lysates

IB: $\alpha$-His

IB: $\alpha$-Flag

IB: $\alpha-\mathrm{HA}$
D
Cell lysates

IB: $\alpha-P P 2 A c$

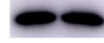

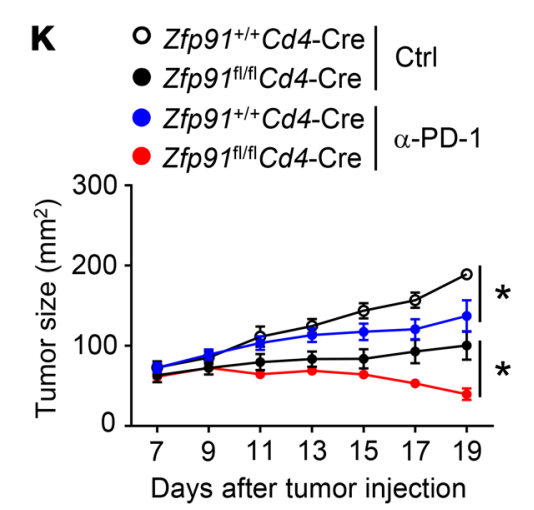

WT KO

IP: $\alpha-P P 2 A a$

IB: $\alpha-P P 2 A c$

Cell lysates

IB: $\alpha$-PP2Aa

IB: $\alpha$-PP2AC

IB: $\beta$-actin
H

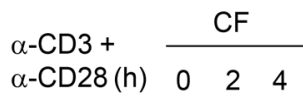

IP: $\alpha$-ZFP91

IB: $a-P P 2 A c$

PP2Ac

$\beta$-actin
I

— US

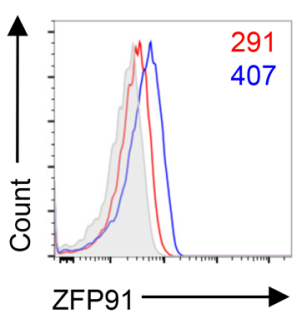

J

O OT-I (MC38)

O OT-I (MC38-OVA)

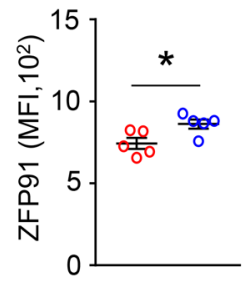

L

Zfp91/++Cd4-Cre
- Zfp91 $91^{\text {ll/fl}} C d 4-C r e$

- Zfp91/++Cd4-Cre $\mid \alpha-P D-1$

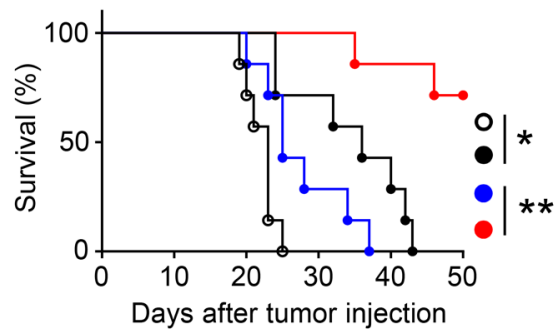

Figure 8. ZFP91 enforces PP2A holoenzyme assembly. (A) IB analysis of ubiquitinated PP2Ac in HEK293T cells transfected with the indicated vectors. (B) IB analysis of PP2Ac K63-linked ubiquitination in Zfp91+/ Cd4-Cre (WT) and Zfp91/l/fl Cd4-Cre (KO) CD90.2+ T cells stimulated with anti-CD3 and anti-CD28 antibodies for 4 hours. (C) Lysates from HEK293T cells transfected with the indicated vectors were subjected to IP. (D) Lysates from WT and KO CD90.2+ T cells stimulated with anti-CD3 and anti-CD28 antibodies for 4 hours were subjected to IP. (E) Lysates from WT CD90.2 $2^{+}$cells stimulated with anti-CD3 and anti-CD28 antibodies were subjected to IP. (F and G) IB analysis of the indicated proteins in whole-cell lysates (WL), cytoplasmic fractions (CF), and nuclear fractions (NF) of WT CD90.2 $2^{+}$cells stimulated with anti-CD3 and anti-CD28 antibodies. (H) IB and IP assays using the cytoplasmic fractions of WT T cells stimulated with anti-CD3 and anti-CD28 antibodies. (I) Flow cytometric analysis of ZFP91 level in T cells stimulated with anti-CD3 and antiCD28 antibodies for 24 hours. Isotype control results are shown in gray shading. US, unstimulated; ST, stimulated. (J) Flow cytometric analysis of ZFP91 expression in OT-I cells in tumors of WT mice given an i.v. injection of $2 \times 10^{6}$ OT-I cells on day 7 after s.c. injection of MC38 cancer cells and MC38-OVA

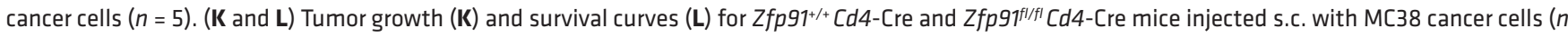
$=7$ ), followed by i.p. injection of anti-PD-1 antibody on days 7, 10, and 13. Ctrl, control antibodies. Data are representative of 3 independent experiments and are presented as the mean \pm SEM. ${ }^{*} P<0.05$ and ${ }^{* *} P<0.01$, by 2 -tailed Student's $t$ test (J and $\mathbf{K}$ ) and log-rank (Mantel-Cox) test (L).

ZFP91-sufficient T cells displayed similar levels of effector cytokine production and Ki-67 expression after 2-DG treatment (Figure 5, G and H). Therefore, ZFP91 restricts glycolytic metabolism to restrain $T$ cell activity.

ZFP91 inhibits mTORC1-dependent metabolic reprogramming. Given that mTOR signaling is involved in metabolic programs to modulate T cell activity (10), we sought to determine whether ZFP91 regulates the activity of the mTOR signaling pathway. Interestingly, immunoblot (IB) and flow cytometric analysis showed increased phosphorylation of ribosomal protein S6, ribosomal protein S6 kinase 1 (S6K1), and the translation initiation inhibitor 4EBP1 in ZFP91-deficient T cells after TCR and CD28 stimulation (Figure 6, A-C), suggesting that ZFP91 inhibits mTORC1 activation. However, mTORC2 activity (AKT phosphorylation at Ser473) in ZFP91-sufficient T cells was unaltered (Figure 6A). To determine the contribution of elevated mTORC1 to ZFP91-deficient T cell activity, we treated ZFP91-sufficient $T$ cells with rapamycin to lower mTORC1. Rapamycin treatment reduced the extracellular acidification rate and erased the differences between ZFP91-deficient and ZFP91-sufficient T cells (Figure 6D). Rapamycin dimin- 
ished effector cytokine production and Ki-67 expression, and rapamycin-treated ZFP91-deficient and ZFP91-sufficient T cells showed comparable levels of effector cytokine production and Ki-67 expression (Figure 6, E and F). We further generate $Z f p 91^{f / f l}$ Raptor $^{f / f l}$ Cd4-Cre mice to confirm the role of elevated mTORC1 in ZFP91-deficient T cell activity. Compared with stimulated $Z f p 91^{f / f l}$ Cd4-Cre T cells, which showed increased glycolytic rates, stimulated T cells from $Z f p 91^{f l / f l}$ Raptor ${ }^{f / f l} \mathrm{Cd}$ 4-Cre mice displayed markedly decreased glycolytic rates (Figure 6G). Importantly, Raptor deficiency eliminated the differences in glycolytic rates between ZFP91-deficient T cells and ZFP91-sufficient T cells (Figure 6G). Consistent with that finding, stimulated $Z f p 91^{f l / f l}$ Raptor ${ }^{f l / f l} C d 4$ Cre $\mathrm{T}$ cells had less effector cytokine production and lower Ki-67 expression than did stimulated $Z f p 91^{f / f l} C d 4$-Cre T cells (Figure 6, $\mathrm{H}$ and I). These results indicate that ZFP91 restrains T cell glycolysis in an mTORC1-dependent manner.

ZFP91 sustains PP2A activity in T cells. PP2A is involved in the regulation of $\mathrm{mTORC} 1$ activity in T cells $(12,13)$. Mass spectrometric analysis has indicated an interaction between ZFP91 and PP2Ac (29). When we coexpressed ZFP91 and PP2Ac in HEK293T cells, the ZFP91-PP2Ac interaction was detectable (Supplemental Figure 5A). In addition, we observed endogenous ZFP91-PP2Ac association in stimulated $\mathrm{T}$ cells (Supplemental Figure 5B). To examine whether ZFP91 regulates PP2A activity in $\mathrm{T}$ cells, we treated $\mathrm{T}$ cells with sphingomyelinase (SMase), which induces ceramide production (12), to activate PP2A phosphatase activity. Treatment of WT T cells with SMase markedly diminished the phosphorylation of S6 (Figure 7A). Moreover, SMase treatment lowered the extracellular acidification rate (ECAR) in WT T cells (Figure 7B). SMase also reduced the levels of cell size and Ki-67 expression of stimulated WT T cells (Figure 7, C and D). However, SMase did not alter S6 phosphorylation levels, glycolytic rates, cell size, or Ki-67 expression in stimulated ZFP91-deficient T cells (Figure 7, A-D). Furthermore, pharmacological inhibition of PP2A with LB-100 increased S6 phosphorylation, the ECAR, cell size, and Ki-67 expression in stimulated WT T cells (Figure 7, E-H). By contrast, ZFP91-deficient $\mathrm{T}$ cells treated or not with LB-100 displayed no apparent differences in $\mathrm{S} 6$ phosphorylation, maximum glycolytic rates, cell size, or Ki-67 expression (Figure 7, E-H). Therefore, ZFP91 is indispensable for PP2A activity in T cells.

To confirm the functional importance of ZFP91 in T cell PP2A activity, we transduced activated WT and ZFP91-deficient $\mathrm{T}$ cells with or without PP2Ac (Figure 7I). PP2Ac overexpression in WT T cells led to the inhibition of mTORC1 activity, coupled with lower glycolytic rates, cell size, and Ki-67 expression levels (Figure 7, J-M). However, PP2Ac overexpression in ZFP91-deficient T cells did not affect the abundance of phosphorylated S6 (p-S6), glycolytic rates, cell size, or Ki-67 expression (Figure 7, J-M). Therefore, ZFP91 is indispensable for PP2A function for inhibition of mTORC1-dependent glycolysis in T cells.

ZFP91 is required for PP2A complex assembly in T cells. ZFP91 acts as an E3 Ub ligase and promotes protein ubiquitination (19). When we induced coexpression of ZFP91 and PP2Ac with HA-tagged Ub in HEK293T cells, we detected ubiquitination of PP2Ac (Figure 8A). However, ZFP91-triggered ubiquitination of PP2Ac was not detectable in the presence of K48-Ub (Figure
8A). On the other hand, overexpression of ZFP91 with K63-Ub but not K63R-Ub induced strong ubiquitination of PP2Ac (Figure 8A). Moreover, ZFP91 deficiency reduced the extent of the K63linked ubiquitination of PP2Ac in activated T cells (Figure 8B), indicating that ZFP91 mediates PP2Ac K63-linked ubiquitination. Since K63-linked Ub chain formation is a signal for protein-protein interactions (30), we next determined the role of ZFP91 in PP2A complex assembly. Overexpression of ZFP91 enhanced the interaction between PP2Aa and PP2Ac in HEK293T cells (Figure 8C). Consistently, the amount of PP2Aa-associated PP2Ac protein was dramatically decreased in stimulated ZFP91-deficient T cells (Figure 8D). Thus, ZFP91 in T cells mediated PP2Ac K63linked ubiquitination to facilitate PP2A complex assembly. Notably, our co-IP assay showed that the interaction between ZFP91 and PP2Ac was strengthened after TCR stimulation (Figure 8E). Furthermore, ZFP91 was predominantly localized in the nuclear fraction in resting $\mathrm{T}$ cells (Figure $8 \mathrm{~F}$ ). Upon TCR stimulation, ZFP91 rapidly translocated from the nucleus to the cytoplasm (Figure 8, F and G). A co-IP assay using cytosolic fractionation revealed that the ZFP91-PP2Ac interaction occurred in the cytoplasm and that the TCR-induced cytosolic translocation of ZFP91 promoted ZFP91-PP2Ac association (Figure 8H). These data suggest that TCR-triggered ZFP91 cytosolic translocation promotes PP2Ac ubiquitination and PP2A complex formation to sustain PP2A phosphatase activity.

We next interrogated the expression profile of ZFP91 in conventional $\mathrm{T}$ cells in different conditions. Naive T cells expressed a lower level of ZFP91 protein, but T cell receptor engagement greatly induced ZFP91 expression (Figure 8I). To detect the induction of ZFP91 in tumor-infiltrating T cells, we injected OT-I CD $8^{+} \mathrm{T}$ cells into the tumor-bearing mice inoculated with both MC38 and MC38-OVA cancer cells. Compared with OT-I $\mathrm{CD}^{+} \mathrm{T}$ cells from MC38 tumors, OT-I CD8 ${ }^{+} \mathrm{T}$ cells from MC38OVA tumors had higher levels of ZFP91 (Figure 8J), suggesting a tumor antigen-specific induction of ZFP91 in tumor-infiltrating T cells. Given that ZFP91 is induced in the TME and that ZFP91 suppresses $\mathrm{T}$ cell antitumor function, we hypothesized that targeting ZFP91 in T cells could improve the antitumor immune response to checkpoint blockade. As expected, the combination of anti-programmed cell death 1 (anti-PD-1) treatment and ZFP91 deletion in $\mathrm{T}$ cells efficiently inhibited tumor progression, as shown by the size of MC38 tumors and tumor-induced lethality (Figure 8, $\mathrm{K}$ and L). These results suggest that targeting ZFP91 in $\mathrm{T}$ cells may be an approach for promoting antitumor responses and improving tumor immunotherapy.

\section{Discussion}

The data presented here demonstrate that ZFP91-dependent PP2A complex assembly disrupted the T cell metabolic pathway and effector function. ZFP91 deficiency impaired PP2A activity and resulted in excessive mTORC1 activity and hyperglycolysis in $\mathrm{T}$ cells, thereby potentiating $\mathrm{T}$ cell activation and proliferation and boosting antitumor $\mathrm{T}$ cell immune responses. In response to TCR stimulation, ZFP91 rapidly translocated from the nucleus to the cytoplasm, which promoted ZFP91PP2Ac interaction and PP2A complex formation in the cytoplasm. Importantly, impairments in $\mathrm{T}$ cell activation and prolif- 
eration correlated with ZFP91 in CRC. These results identified a ZFP91-governed mechanism disturbing the $\mathrm{T}$ cell metabolic program and antitumor function in the TME.

$\mathrm{T}$ cell functional impairment in the TME is a hallmark of many cancers $(31,32)$. T cell dysfunction is generally associated with disrupted metabolic pathways, impaired effector functions, and multiple overexpressed inhibitory receptors $(9,31)$. Although checkpoint blockade immunotherapies targeting the inhibitory receptors have yielded clinical benefits, only a fraction of patients achieve sustained clinical responses (33). The molecular mechanisms controlling $\mathrm{T}$ cell functional defects in the TME need to be further determined. Using previously published scRNA-Seq data (22), we observed that the impairments in T cell activation and proliferation correlated with ZFP91 in CRC tissue samples. Correspondingly, $\mathrm{T}$ cell-specific deletion of $Z f p 91$ led to enhanced $\mathrm{T}$ cell proliferation and potentiated $\mathrm{T}$ cell antitumor function in mouse cancer models. Our transcriptomic analysis revealed that loss of ZFP91 augmented the expression of genes involved in T cell activation, proliferation, and glycolysis. In activated $\mathrm{T}$ cells, ZFP91 deficiency increased mTORC1 activity to increase glycolytic activity, thereby supporting $\mathrm{T}$ cell expansion and antitumor functions. These results identify ZFP91 as a metabolic regulator that perturbs $\mathrm{T}$ cell metabolic and functional states in the TME.

$\mathrm{PP} 2 \mathrm{~A}$ is the assembly product of the PP2Aa, PP2Ab, and PP2Ac subunits and regulates $\mathrm{T}$ cell metabolism and effector function $(13,14,34,35)$; however, how TCR stimulation directs the holoenzyme assembly of PP2A remains unclear. Our pharmacological experiments showed that ZFP91 was required for PP2A phosphatase activity to restrict mTORC1-mediated $\mathrm{T}$ cell glycolysis and proliferation. ZFP91 triggered PP2Ac K63-linked ubiquitination, which provided a signal for PP2A complex assembly. Notably, ZFP91 was predominantly localized in the nucleus in resting T cells. Upon TCR stimulation, ZFP91 rapidly translocated from the nucleus to the cytoplasm and then promoted ZFP91-PP2Ac association and PP2A complex formation in the cytoplasm. On the basis of these data, we propose that TCR-induced ZFP91 cytosolic translocation initiates PP2A holoenzyme assembly to perturb $\mathrm{T}$ cell metabolic and functional fitness.

ZFP91 is a potential oncogenic protein expressed in various tumor types and is required for tumorigenesis or tumor metastasis $(20,21)$. We found that ZFP91 in T cells diminished T cell proliferation and dampened antitumor $\mathrm{T}$ cell immune responses. Together with previous reports, our current study suggests that targeting ZFP91 may not only suppress tumorigenesis or tumor metastasis but also boost antitumor $\mathrm{T}$ cell responses, and thus has important clinical applications. Indeed, it is still unclear how TCR signaling triggers the cytosolic translocation of ZFP91 as well as the induction of ZFP91. Therefore, the upstream signals that control the induction and cytosolic translocation of ZFP91 in T cells need to be further studied.

In summary, our findings identify a ZFP91-dependent mechanism that sustains the holoenzyme assembly of PP2A to restrict $\mathrm{T}$ cell metabolic and functional fitness. Our study not only defines ZFP91-mediated T cell glycolytic and functional defects in mouse tumor models, but also highlights the correlation between $\mathrm{T}$ cell dysfunction and ZFP91 in CRC. Our results also suggest that targeting ZFP91 provides a promising strategy for the improvement of cancer immunotherapy.

\section{Methods}

Human CRC samples. Human CRC tissues were obtained at Ruijin Hospital in Shanghai, China. Solid cancer tissues were freshly isolated and digested. TILs were enriched by density-gradient centrifugation. $\mathrm{T}$ cells were purified using the REAlease CD4/8 MicroBead Kit (130121-561, Miltenyi Biotec).

Mice. Zfp91-floxed mice on a C57BL/6 background were generated using a LoxP-targeting system at the Shanghai Model Organisms Center Inc. The $Z f p 91$-floxed mice were crossed with $C d 4$-Cre transgenic mice (The Jackson Laboratory) on a B6 background to obtain age-matched $Z f p 91^{+/+} C d 4$-Cre and $Z f p 91^{f / f l} C d 4$-Cre mice for experiments. B6.SJL mice, OT-I TCR-transgenic mice, and OT-II TCR-transgenic mice on a C57BL/6 background were purchased from The Jackson Laboratory. The OT-I mice and OT-II mice were crossed with $Z f p 91^{f / / f l} \mathrm{Cd}$-Cre mice to produce $Z f p 91^{f / / f l} C d 4$-Cre OT-I mice and $Z f p 91^{f / f l} C d 4$-Cre OT-II mice. The Raptor-floxed mice on a C57BL/6 background were purchased from The Jackson Laboratory and crossed with $Z f p 91^{f / f l} \mathrm{Cd}$-Cre mice to obtain $Z f p 91^{f / f l}$ Raptor ${ }^{f / f l} C d 4$-Cre mice. Mice were randomly allocated to each experimental group. All mice used in the experiments were age and sex matched, and all mice were maintained in a pathogen-free facility.

Plasmids, antibodies, and reagents. Mouse ZFP91, PP2Aa, and PP2Ac genes were amplified from the mouse tissue cDNA library and subsequently subcloned into the lentivirus vector pLVX-IRES-ZsGreen1. HA-tagged K48-, K63-, and K63R-mutant Ub were subcloned into the pcDNA3-HA vector. Antibodies against p-S6 (4858s; 1:1000 for WB), PP2Aa (2041s; 1:1000 for Western blotting [WB]), PP2Ac (2038s; 1:1000 for WB), p-Akt (Ser473) (4060s; 1:1000 for WB), p-4EBP1 (Ser65) (9451s; 1:1000 for WB), p-p70 S6 kinase (Thr389) (9234s; 1:1000 for WB), and K63 polyubiquitin (D7A11; 1:1000 for WB) were purchased from Cell Signaling Technology. HRP-conjugated anti-HA antibody (3F10; 1:2000 for WB) was purchased from Roche. Anti-Flag (M2, F3165; 1:2000 for WB) and $\beta$-actin (A2228100UL; 1:5000 for WB) antibodies were from MilliporeSigma. AntiHis antibody (D291-3; 1:1000 for WB) was obtained from Medical and Biological Laboratories. Anti-ZFP91 antibody (A303-245A; 1:1000 for WB) was purchased from Bethyl Laboratories. Anti-lamin B antibody (66095-1-Ig; 1:1000 for WB) was purchased from Proteintech. For flow cytometric experiments, antibodies against CD4 (GK1.5), CD8 (53-6.7), CD44 (IM7), CD62L (MEL-14), Ki-67 (So1A15), IFN- $\gamma$ (XMG1.2), IL-17 (eBio17B7), and Foxp3 (FJK-16s) were purchased from Thermo Fisher Scientific. p-S6 ribosomal protein (Ser235/236) (D57.2.2E) and XP Rabbit monoclonal antibody (APC Conjugate) were purchased from Cell Signaling Technology. Anti-Glut1 antibody was purchased from Abcam (ab115730). For fluoresence staining, IFKine Red Donkey Anti-mouse IgG secondary antibody was purchased from Abbkine (A24411-1). Anti-mouse PD-1 (clone J43) and isotype control IgG (hamster IgG) antibodies were from Bio X Cell. SMase from Staphylococcus aureus (S8633) was purchased from MilliporeSigma. LB-100 (S7537) was purchased from Selleck Chemicals.

Flow cytometry. Freshly isolated cells were resuspended in PBS containing $2 \%$ FBS with the indicated fluorochrome-conjugated antibodies for surface staining. TILs were enriched by density-gradient centrifugation and stimulated with $50 \mathrm{ng} / \mathrm{mL}$ PMA and $500 \mathrm{ng} / \mathrm{mL}$ ionomycin in the presence of monensin for 5 hours. After stimulation, cells were stained with anti-CD8 or anti-CD4 first, and then fixed, permeabilized, and stained with the indicated antibodies for intracellular cytokine 
expression analysis. Stimulated T cells were treated with Fix Buffer I (BD Biosciences) at $37^{\circ} \mathrm{C}$ for 10 minutes, incubated in pre-cold Perm Buffer III (BD Biosciences) for another 20 minutes, and then stained with p-S6 antibody for flow cytometric analysis. For cell proliferation analysis, isolated T cells were labeled with $5 \mu \mathrm{M}$ CFSE, cultured with irradiated splenocytes depleted of $\mathrm{T}$ cells, and stimulated with plate-bound antiCD3 $(1 \mu \mathrm{g} / \mathrm{mL})$ and anti-CD28 $(1 \mu \mathrm{g} / \mathrm{mL})$ for 72 hours. For Ki-67 detection, stimulated $\mathrm{T}$ cells were fixed, permeabilized, and stained with the indicated antibodies according to the manufacturer's protocol (Thermo Fisher Scientific).

Tcellpurification and culturing. Peripheral $\mathrm{CD}^{+}, \mathrm{CD} 8^{+}$, or CD90. $2^{+} \mathrm{T}$ cells were isolated from mouse spleens and lymph nodes by positive-selection magnetic beads (Miltenyi Biotec). The cells were then stimulated with plate-bound anti-CD3 $(1 \mu \mathrm{g} / \mathrm{mL})$ and anti-CD28 $(1 \mu \mathrm{g} / \mathrm{mL})$ antibodies followed by culturing with complete RPMI 1640 medium containing $10 \%$ FBS and antibiotics. T cells $\left(2 \times 10^{5}\right.$ per well $)$ in 96 -well plates were stimulated for flow cytometric analysis. T cells $\left(5 \times 10^{6}\right.$ per well $)$ in 6-well plates were stimulated for Seahorse, RNA-Seq, IB, and IP analyses.

TIL isolation. Tumors from tumor-bearing mice were collected, cut into 1-3 mm-sized pieces, and digested for 30 minutes in RPMI 1640 medium containing $10 \% \mathrm{FBS}, 0.2 \mathrm{mg} / \mathrm{mL}$ collagenase IV, and $20 \mu \mathrm{g} / \mathrm{mL}$ DNase I. Digested tissues were filtered through a $100 \mu \mathrm{m}$ cell strainer and washed with PBS. Percoll $(40 \%, 4 \mathrm{~mL})$ was added to resuspend tissues, and $2.5 \mathrm{~mL}$ 70\% Percoll was added slowly from the bottom of tube. Centrifugation was performed at $587 \mathrm{~g}$ for $20 \mathrm{~min}$ utes at an acceleration of 1 and a deceleration of 1 . Lymphocytes were enriched at the bilayer interface and washed with PBS to obtain TILs.

ELISA and quantitative RT-PCR. Supernatants of in vitro cell cultures were analyzed by ELISA using a commercial assay system from Thermo Fisher Scientific. Total RNA was extracted from isolated human tumor-infiltrating $\mathrm{T}$ cells and subjected to quantitative reverse transcriptase PCR (qRT-PCR) using gene-specific primers. Data were generated using the comparative threshold cycle $(\Delta \mathrm{Ct})$ method with normalization to the reference gene $A c t b$. The following primer sequences were used in the studies: Zfp 91 forward, GCCATCGTGATACAGAGAACACC, $Z f p 91$ reverse, CTAATGCCACCTGGAGACTGATG; Mki67 forward, GAAAGAGTGGCAACCTGCCTTC, Mki67 reverse, GCACCAAGTTTTACTACATCTGCC; Pttg1 forward, GCTTTGGGAACTGTCAACAGAGC, Pttg1 reverse, CTGGATAGGCATCATCTGAGGC; Ccnb1 forward, GACCTGTGTCAGGCTTTCTCTG, Ccnb1 reverse, GGTATTTTGGTCTGACTGCTTGC; Tyms forward, GGTGTTTTGGAGGAGTTGCTGTG, Tyms reverse, GGAGAATCCCAGGCTGTCCAAA; Tbx21 forward, ATTGCCGTGACTGCCTACCAGA, Tbx21 reverse, GGAATTGACAGTTGGGTCCAGG; Gzma forward, CCACACGCGAAGGTGACCTTAA, Gzma reverse, CCTGCAACTTGGCACATGGTTC; Ifng forward, GAGTGTGGAGACCATCAAGGAAG, Ifng reverse, TGCTTTGCGTTGGACATTCAAGTC; Prfl forward, ACTCACAGGCAGCCAACTTTGC, Prf1 reverse, CTCTTGAAGTCAGGGTGCAGCG; and Actb forward, CACCATTGGCAATGAGCGGTTC, Actb reverse, AGGTCTTTGCGGATGTCCACGT.

Tumor model. MC38 murine colon cancer cells (Kerafast) and MC38-OVA murine colon cancer cells (generated in-house), and B16-F10 murine melanoma cells (from the American Type Culture Collection [ATCC]) all tested negative for mycoplasma contamination. All cell lines used in this study had been previously authenticated. MC38 murine colon cancer cells and MC38-OVA murine colon cancer cells were cultured in DMEM supplemented with 10\% FBS. B16-F10 murine melanoma cells were cultured with complete RPMI-1640 medium containing $10 \%$ FBS. These tumor cells $\left(5 \times 10^{5}\right.$ cells $)$ were s.c. injected into 6- to 8-week-old mice. Tumor size was calculated as the length times the width. Mice bearing a tumor with a size that was larger than $225 \mathrm{~mm}^{2}$ were euthanized. Tumor size and survival were recorded from day 7 after tumor injection. To detect the induction of ZFP91 in tumor-infiltrating T cells, MC38 $\left(5 \times 10^{5}\right.$ cells $)$ and MC38OVA $\left(5 \times 10^{5}\right.$ cells $)$ cancer cells were s.c. injected into the left and right flanks of C57BL/6 mice. OT-I CD8 ${ }^{+} \mathrm{T}$ cells $\left(2 \times 10^{6}\right)$ were i.v. injected into tumor-bearing mice on day 7 after tumor cell injection. On day 14, TILs from MC38 and MC38-OVA tumor tissues were collected for further analysis. For cotransfer experiments, $Z f p 91^{+/+} C d 4$-Cre OT-I and $Z f p^{9} 91^{f / f l} C d 4$-Cre OT-I cells were stimulated with $250 \mathrm{ng} / \mathrm{mL}$ OVA $_{257-264}$ peptides plus $10 \mathrm{ng} / \mathrm{mL} \mathrm{IL-2} \mathrm{for} 2$ days and then cultured in fresh medium containing $10 \mathrm{ng} / \mathrm{mL} \mathrm{IL-2} \mathrm{for} \mathrm{the} \mathrm{other} 2$ days. $Z f p 91^{+/+}$ Cd4-Cre (WT) OT-I cells were labeled with CFSE $(5 \mu \mathrm{M})$ and $Z f p 91^{f / f / l}$ Cd4-Cre (KO) OT-I cells were labeled with CellTrace Violet (CTV, 5 $\mu \mathrm{M}$, Invitrogen). CFSE-labeled WT OT-I cells and CTV-labeled KO OT-I cells were mixed at a 1:1 ratio $\left(5 \times 10^{6}\right.$ cells each $)$ and adoptively transferred into MC38-OVA tumor-bearing mice on day 7 after tumor injection. Tumor tissues and spleens were harvested for subsequent experiments on day 7 after OT-I cell transfer.

Lentivirus transduction. HEK293T cells were transfected with lentivirus expression vectors and packing vectors to produce lentivirus particles. CD 90.2 $2^{+} \mathrm{T}$ cells from $Z f p 91^{+/+} C d 4$-Cre and $Z f p 91^{f / f l} \mathrm{Cd}$-Cre mice were stimulated with anti-CD3 $(1 \mu \mathrm{g} / \mathrm{mL})$ and anti-CD28 $(1 \mu \mathrm{g} /$ $\mathrm{mL}$ ) antibodies for 24 hours, followed by infection with lentiviruses in fresh medium containing IL-2 (10 ng/mL), $\beta$-mercaptoethanol (50 $\mu \mathrm{M})$, and polybrene $(6 \mu \mathrm{g} / \mathrm{mL})$. Transduced cells were enriched on the basis of the expression of GFP after 3 days.

RNA-Seq analysis. WT and ZFP91-deficient (KO) CD90.2 $2^{+} \mathrm{T}$ cells were isolated from the spleens of tumor-free $Z f p 91^{+/+} C d 4$-Cre and $Z f p$ $91^{f / f l} \mathrm{Cd} 4$-Cre mice or the MC38 tumors of tumor-bearing $Z f \mathrm{p} 91^{+/+} \mathrm{Cd} 4$ Cre and $Z f p 91^{f / f l} C d 4$-Cre mice. Naive CD $90.2^{+} \mathrm{T}$ cells were stimulated with 6-well, plate-bound anti-CD3 $(1 \mu \mathrm{g} / \mathrm{mL})$ and anti-CD28 $(1 \mu \mathrm{g} / \mathrm{mL})$ antibodies for 24 hours. Total RNA was extracted from tumor-infiltrating T cells or in vitro-activated T cells. Isolated total RNA was subjected to RNA-Seq using the Illumina Nextseq 500 (75 bp paired-end reads). The raw reads were aligned to the mouse reference genome (version mm10) using Tophat2 RNASeq alignment software. The mapping rate was approximately $96 \%$ overall across all the samples in the data set. The raw reads were processed to generate read counts for every gene. The read counts were normalized using R package DESeq2, and were then centered and scaled for each gene, generating $z$ scores. $P$ values obtained from multiple tests were adjusted using Benjamini-Hochberg correction. The RNA-Seq data reported here are available in the NCBI's Trace and Short-Read Archive (accession number PRJNA646554).

Glycolytic and mitochondrial respiration rate measurement. Peripheral CD4 $4^{+}, \mathrm{CD}^{+}$, or CD90.2 $2^{+} \mathrm{T}$ cells were stimulated with anti-CD3 and anti-CD28 antibodies for 24 hours and plated at $2 \times 10^{5}$ cells per well in a 96-well Seahorse assay plate. ECAR and oxygen consumption rate (OCR) measurements were conducted with the Seahorse XFe96 Extracellular Flux Analyzer (Aglient Technologies). ECAR ( $\mathrm{mpH} / \mathrm{min}$ ) and OCR ( $\mathrm{pmol} / \mathrm{min}$ ) were measured as indicated upon cell treatment with glucose $(10 \mathrm{mM})$, oligomycin $(2 \mu \mathrm{M}), 2-\mathrm{DG}(50 \mathrm{mM})$, carbonyl cyanide 4-(trifluoromethoxy)phenylhydrazone (FCCP) $(1 \mu \mathrm{M})$, and rotenone/ antimycin A $(0.5 \mu \mathrm{M})$ according to the manufacturers' instructions (all 
products from Agilent Technologies). The XF Cell Mito and the XF Glycolytic stress test kits were purchased from Agilent Technologies.

IB and ubiquitination assays. Stimulated T cells were rinsed once with ice-cold PBS and lysed with RIPA buffer containing a protease inhibitor on ice for 30 minutes. The cell lysates were clarified by centrifugation at $18,407 \mathrm{~g}$ for 10 minutes. For IPs, the supernatants were immunoprecipitated with the indicated antibodies overnight. The protein $G$ agarose beads were washed 3 times with lysis buffer followed by incubation with the supernatants for another 2 hours. After incubation, the immunoprecipitated proteins were washed 3 times with lysis buffer. The samples were denatured by the addition of sample buffer and boiling for $10 \mathrm{~min}$ utes, resolved by $8 \%-16 \%$ SDS-PAGE, and analyzed by immunoblotting. For ubiquitination assays, immunoprecipitated PP2Ac from stimulated T cells was detected by immunoblotting using Ub antibodies.

Statistics. Statistical analysis was performed using GraphPad Prism, version 8.0 (GraphPad Software). A 2-tailed, unpaired Student's $t$ test was performed as indicated in the figure legends. Survival curves were analyzed by log-rank (Mantel-Cox) test. Data are presented as the mean \pm SEM. A $P$ value of less than 0.05 was considered statistically significant.

Study approval. The collection of human CRC samples was approved by the Clinical Research Ethics Committee of Ruijin Hospital and complied with all relevant ethics regulations (2019-205). All animal procedures were performed according to protocols approved by the IACUC of the Shanghai Jiao Tong University School of Medicine.

\section{Author contributions}

FW performed the experiments, analyzed the data, and wrote the manuscript. YZ, XY, XLT, RD, ZH, and AW helped with mouse experiments and flow cytometry and analyzed the data. ZW, YY, and QZ designed the experiments, interpreted the results, wrote the manuscript, and oversaw the research project.

\section{Acknowledgments}

This study was supported by grants from the National Natural Science Foundation of China (81930040, 31922025, 31870884), the National Key Research and Development Program of China (2020YFA0803603), and the Shanghai Science and Technology Commission (20JC1410100).

Address correspondence to: Qiang Zou or Youqiong Ye, Shanghai Institute of Immunology, Department of Immunology and Microbiology, Shanghai Jiao Tong University School of Medicine, 280 South Chongqing Road, Shanghai 200025, China. Phone: 086.021.64666150; Email: Qzou1984@sjtu.edu.cn (QZ). Phone: 086.021.63846590; Email: youqiong.ye@shsmu.edu.cn (YY). Or to: Zhengting Wang, Department of Gastroenterology, Ruijin Hospital, Shanghai Jiao Tong University School of Medicine, 197 Ruijin Er Road, Shanghai 200025, China. Phone: 086.021.64370045; Email: zhengtingwang@shsmu.edu.cn.
1. Ma X, et al. Cholesterol induces $\mathrm{CD}^{+} \mathrm{T}$ cell exhaustion in the tumor microenvironment. Cell Metab. 2019;30(1):143-156.

2. Wei J, et al. Targeting REGNASE-1 programs long-lived effector $\mathrm{T}$ cells for cancer therapy. Nature. 2019;576(7787):471-476.

3. Bantug GR, et al. The spectrum of T cell metabolism in health and disease. Nat Rev Immunol. 2018;18(1):19-34.

4. Chapman NM, et al. Metabolic coordination of T cell quiescence and activation. Nat Rev Immunol. 2020;20(1):55-70.

5. Geiger R, et al. L-Arginine modulates T cell metabolism and enhances survival and anti-tumor activity. Cell. 2016;167(3):829-842.

6. Rivadeneira DB, Delgoffe GM. Antitumor T-cell reconditioning: improving metabolic fitness for optimal cancer immunotherapy. Clin Cancer Res. 2018;24(11):2473-2481.

7. Siska PJ, Rathmell JC. T cell metabolic fitness in antitumor immunity. Trends Immunol. 2015;36(4):257-264.

8. Hope HC, Salmond RJ. Targeting the tumor microenvironment and $\mathrm{T}$ cell metabolism for effective cancer immunotherapy. Eur J Immunol. 2019;49(8):1147-1152.

9. Kishton RJ, et al. Metabolic regulation of T cell longevity and function in tumor immunotherapy. Cell Metab. 2017;26(1):94-109.

10. Huang $\mathrm{H}$, et al. mTOR signaling at the crossroads of environmental signals and T-cell fate decisions. Immunol Rev. 2020;295(1):15-38.

11. Yu Q, et al. SIRT1 and HIF1 $\alpha$ signaling in metabolism and immune responses. Cancer Lett. 2018;418:20-26.

12. Apostolidis SA, et al. Phosphatase PP2A is requisite for the function of regulatory T cells. Nat Immunol. 2016;17(5):556-564.
13. Eil R, et al. Ionic immune suppression within the tumour microenvironment limits $\mathrm{T}$ cell effector function. Nature. 2016;537(7621):539-543.

14. Delgoffe GM. PP2A's restraint of mTOR is critical for T(reg) cell activity. Nat Immunol. 2016;17(5):478-479.

15. Kingwell K. Cancer: live screening of immunotherapy targets. Nat Rev Drug Discov. 2014;13(4):258.

16. Zhou P, et al. In vivo discovery of immunotherapy targets in the tumour microenvironment. Nature. 2014;506(7486):52-57.

17. Ho WS, et al. Pharmacologic inhibition of protein phosphatase-2A achieves durable immune-mediated antitumor activity when combined with PD-1 blockade. Nat Commun. 2018;9(1):2126.

18. Cho US, Xu W. Crystal structure of a protein phosphatase 2A heterotrimeric holoenzyme. Nature. 2007;445(7123):53-57

19. Jin $X$, et al. An atypical E3 ligase zinc finger protein 91 stabilizes and activates NF-kappaB-inducing kinase via Lys63-linked ubiquitination. J Biol Chem . 2010;285(40):30539-30547.

20. Unoki M, et al. Identification of a novel human gene, ZFP91, involved in acute myelogenous leukemia. Int JOncol. 2003;22(6):1217-1223.

21. $\mathrm{Xu} \mathrm{SH}$, et al. ECD promotes gastric cancer metastasis by blocking E3 ligase ZFP91-mediated hnRNP F ubiquitination and degradation. Cell Death Dis. 2018;9(5):479.

22. Zhang L, et al. Lineage tracking reveals dynamic relationships of $\mathrm{T}$ cells in colorectal cancer. Nature. 2018;564(7735):268-272.

23. Kurtulus S, et al. Checkpoint blockade immunotherapy induces dynamic changes in PD-1 CD8 ${ }^{+}$tumor-infiltrating T cells. Immunity. 2019;50(1):181-194.

24. Lu YC, et al. Single-cell transcriptome analysis reveals gene signatures associated with T-cell persistence following adoptive cell therapy. Cancer Immunol Res. 2019;7(11):1824-1836.

25. Wu TD, et al. Peripheral T cell expansion predicts tumour infiltration and clinical response. Nature. 2020;579(7798):274-278.

26. Zhang L, et al. Single-cell analyses inform mechanisms of myeloid-targeted therapies in colon cancer. Cell. 2020;181(2):442-459.

27. Cancer Genome Atlas Research Network, et al. The cancer genome atlas pan-cancer analysis project. Nat Genet. 2013;45(10):1113-1120.

28. Hu Z, et al. Acylglycerol kinase maintains metabolic state and immune responses of $\mathrm{CD}^{+} \mathrm{T}$ cells. Cell Metab. 2019;30(2):290-302.

29. Hein MY, et al. A human interactome in three quantitative dimensions organized by stoichiometries and abundances. Cell. 2015;163(3):712-723.

30. Swatek KN, Komander D. Ubiquitin modifications. Cell Res. 2016;26(4):399-422.

31. Hashimoto M, et al. CD8 T cell exhaustion in chronic infection and cancer: opportunities for interventions. Annu Rev Med. 2018;69:301-318.

32. Li W, et al. cGAS-STING-mediated DNA sensing maintains $\mathrm{CD} 8^{+} \mathrm{T}$ cell stemness and promotes antitumor T cell therapy. Sci Transl Med. 2020;12(549):eaay9013.

33. Wang G, et al. Multiplexed activation of endogenous genes by CRISPRa elicits potent antitumor immunity. Nat Immunol. 2019;20(11):1494-1505.

34. Yu X, et al. Metabolic control of regulatory T cell stability and function by TRAF3IP3 at the lysosome. J Exp Med. 2018;215(9):2463-2476.

35. Xu Q, et al. Phosphatase PP2A is essential for $\mathrm{T}_{\mathrm{H}} 17$ differentiation. Proc Natl Acad Sci US A. 2019;116(3):982-987. 